ULB-TH/08-11

UB-ECM-PF-08-10

\title{
Extended Symmetries in Supergravity: the Semi-simple Case
}

\author{
Axel Kleinschmidt ${ }^{1}$ and Diederik Roest ${ }^{2}$ \\ ${ }^{1}$ Physique Théorique et Mathématique, \\ Université Libre de Bruxelles \& International Solvay Institutes, \\ Boulevard du Triomphe, ULB - CP 231, B-1050 Bruxelles, Belgium \\ 2 Departament Estructura i Constituents de la Materia \\ \& Institut de Ciències del Cosmos, \\ Universitat de Barcelona, \\ Diagonal 647, 08028 Barcelona, Spain \\ E-mails: axel.kleinschmidt@ulb.ac.be, droest@ecm.ub.es
}

\begin{abstract}
The bosonic sector of various supergravity theories reduces to a homogeneous space $G / H$ in three dimensions. The corresponding algebras $\mathfrak{g}$ are simple for (half-)maximal supergravity, but can be semi-simple for other theories. We extend the existing literature on the Kac-Moody extensions of simple Lie algebras to the semi-simple case. Furthermore, we argue that for $\mathcal{N}=2$ supergravity the simple algebras have to be augmented with an $\mathfrak{s u}(2)$ factor.
\end{abstract}




\section{Introduction}

One of the intriguing aspects of supergravities are their hidden symmetries. Upon dimensional reduction over a torus $T^{d}$ of any theory containing gravity, one would expect an $S L(d)$ global symmetry in four or more dimensions and $S L(d+1)$ in three dimensions. These are lower-dimensional remnants of the diffeomorphisms on the internal torus. In addition there will be generators that originate from e.g. higher-dimensional gauge symmetries. The surprising feature of supergravity is that these two types of symmetries combine to form a much larger symmetry group. For instance, for maximal supergravity the global symmetries are the exceptional group $11 E_{11-D}$ in $D \geq 3$ dimensions $[1,2]$.

The phenomenon of hidden symmetries is perhaps most striking in three dimensions. All propagating degrees of freedom can be described by scalars and, in the cases of interest, these transform in a non-linear representation under a global symmetry. That is, the bosonic sector of the theory consists of gravity (which is non-propagating in three dimensions) coupled to a scalar coset $G / H$, where $G$ is the global symmetry group and $H$ is its maximal compact subgroup. For instance, maximal supergravity in three dimensions reduces to the coset

$$
\frac{G}{H}=\frac{E_{8}}{S O(16)}
$$

while half-maximal supergravity is given by

$$
\frac{G}{H}=\frac{S O\left(8,8+n_{\mathrm{V}}\right)}{S O(8) \times S O\left(8+n_{\mathrm{V}}\right)},
$$

where $n_{V}$ corresponds to the number of vector multiplets in ten dimensions.

So far our discussion has been concerned with finite-dimensional hidden symmetries, generated by a simple Lie algebra $\mathfrak{g}$. Yet more intriguing are the results and conjectures on extended symmetries, featuring the infinite-dimensional Kac-Moody extensions of the simple $\mathfrak{g}$. For instance, it has been proven that the affine extension $\mathfrak{g}^{+}$appears upon reduction to two dimensions [3-6]. In particular, all bosonic solutions in $D=2$ form a non-linear representation of $\mathfrak{g}^{+}$. The over- and very-extensions $\mathfrak{g}^{++}$and $\mathfrak{g}^{+++}$have been conjectured to play a role in $D=1$ and $D=0$, respectively $[3,7]$. Finally, further conjectures have been made about the role of the latter two Kac-Moody algebras in relation to the full supergravity without dimensional reduction to $D<2$, see [8-10] and $[11-13]$ respectively.

We will not be concerned with the dynamical realisation of the Kac-Moody symmetries according to these different proposals. Instead, we will focus on a necessary requirement for these conjectures to work: the algebraic correspondence between bosonic supergravity fields and a well-defined truncation of the Kac-Moody generators, and we focus on the very-extended algebras.2 This matching has been performed for the physical degrees of freedom of many supergravities, see e.g. [14-17].

\footnotetext{
${ }^{1}$ The groups and algebras of this paper are of split real form unless explicit compact notation is used.

${ }^{2}$ Our construction of extended algebras in the semi-simple case and the analysis of their spectrum is also valid for the over-extended case.
} 
This correspondence can be extended to non-propagating supergravity fields. More concretely, many supergravities allow for the introduction of certain $(D-1)$ - and $D$ form potentials. The former can be seen as the duals of deformation parameters that introduce massive deformations or gaugings to the theory. The latter can correspond to certain constraints that have to be imposed on the gauge parameters for consistency of the gaugings. These non-propagating degrees of freedom will be referred to as deformation potentials and top-form potentials, respectively. Interestingly, it was found recently that the Kac-Moody algebra $\mathfrak{g}^{+++}$contains exactly the right generators to correspond to the deformation and top-form potentials of maximal and half-maximal supergravity in all dimensions [18-20] (see also [10,21,22] for earlier results in ten dimensions and [23, 24] for a detailed analysis of the algebraic structure in the gauged case).

In this paper we want to address the natural and interesting question to what extent the above results hold for other supergravities as well. We will mainly be concerned with supergravity theories that can be formulated in four or more dimensions 3 , and not those that only live in three dimensions. Nevertheless, as stressed before, it will be crucial for our analysis to understand what the various supergravities reduce to in three dimensions. The bosonic sector of any supergravity reduces to a scalar manifold coupled to gravity, and different amounts of supersymmetry impose different constraints on this scalar manifold [25]. In particular, the bosonic sectors of all supergravity theories with more than eight supercharges, i.e. with $\mathcal{N}>2$, reduce to a homogeneous space $G / H$ in three dimensions. Examples are the maximal and half-maximal supergravities given in (11) and (2), but this result also holds for the 'exceptional' supergravity theories with $10,12,18,20$ or 24 supercharges [26].

For theories with $\mathcal{N} \leq 2$, i.e. with eight or less real supercharges, one encounters more general scalar manifolds than homogeneous ones. Nevertheless, the subset of theories for which the scalar manifold is homogeneous is more tractable and still interesting, and has proven very valuable in many applications. In particular, one can study the hidden symmetries and ask similar questions about the corresponding Kac-Moody extensions as discussed above in the context of maximal and half-maximal supergravity. In this paper we will only be concerned with the theories at these 'points of homogeneity' in the moduli space of $\mathcal{N} \leq 2$ theories.

Two points are important to notice in the context of this paper. The first concerns the algebra $\mathfrak{g}$ that is generated by the group $G$ of isometries of the homogeneous spaces in three dimensions. As can be read off from (11) and (2), this is always a simple algebra for maximal and half-maximal supergravity. In fact this holds for all supergravity theories with $\mathcal{N}>2$. A simple Lie algebra can straightforwardly be promoted to a Kac-Moody algebra by affine, over- and very-extensions $[27,28]$. In contrast, for $\mathcal{N} \leq 2$ the algebra of isometries $\mathfrak{g}$ is not necessarily simple but can be semi-simple as well $4^{4}$ In fact, as we will see in sections 3 and 5 , it can be argued that it is generically semi-simple for homogeneous scalar manifolds in $\mathcal{N}=2$ and $\mathcal{N}=1$ supergravities. One thus needs a

\footnotetext{
${ }^{3}$ For this reason we will adhere to four-dimensional notation for $\mathcal{N}$, i.e. the number of supercharges of a theory is $4 \mathcal{N}$ in any dimension.

${ }^{4}$ There are also (non-symmetric) homogeneous spaces with non-semi-simple groups of isometries, see e.g. [29], but we will not consider these here.
} 
proposal for the corresponding affine, over- and very-extensions in the semi-simple case. This will be provided in section 2 .

\begin{tabular}{||c||c|c||}
\hline$D$ & $H_{\mathrm{R}}$ & with \\
\hline \hline 11 & 1 & $n_{1}+n_{2}=\mathcal{N} / 4$ \\
\hline 10 & $S O\left(n_{1}\right) \times S O\left(n_{2}\right)$ & $n=\mathcal{N} / 4$ \\
\hline 9 & $O(n)$ & $n=\mathcal{N} / 4$ \\
\hline 8 & $U(n)$ & $n=\mathcal{N} / 4$ \\
\hline 7 & $S p(n)$ & $n=\mathcal{N} / 2$ \\
\hline 6 & $S p\left(n_{1}\right) \times S p\left(n_{2}\right)$ & $n_{1}+n_{2}=\mathcal{N} / 2\left(n_{1,2} \leq 2\right)$ \\
\hline 5 & $S p(n)$ & $n=\mathcal{N}$ \\
\hline 4 & $U(n)$ & $n=2 \mathcal{N}$ \\
\hline 3 & $S O(n)$ & \\
\hline
\end{tabular}

Table 1: The $R$-symmetry groups of supergravities for various values of $D$ and $\mathcal{N}$, adapted from [30,31], and where $S p(n)$ denotes the compact symplectic group of dimension $n(2 n+$ 1) (sometimes also denoted in the literature as $U S p(2 n)$ ). In ten dimensions there are two possibilities for maximal supergravity while in six dimensions there are two possibilities for half-maximal supergravity. In both cases the non-chiral theory has $n_{1}=n_{2}$ while the chiral one has $n_{1} n_{2}=0$.

The second point concerns the R-symmetry group, i.e. the global symmetry that rotates the different supercharges of a supersymmetric theory. The R-symmetry groups for different values of $D$ and $\mathcal{N}$ have been summarised in table 1 . It turns out that the R-symmetry group $H_{\mathrm{R}}$ of maximal supergravity coincides with the compact part of the global symmetry group, i.e. we have $H_{\mathrm{R}}=H$. This can be checked for $D=3$ using (11) but holds also in higher dimensions 5 The same is true for half-maximal supergravity if one only considers the graviton multiplet (corresponding to $n_{\mathrm{V}}=-7$ in (2)). In the presence of additional vector multiplets (or tensor multiplets in the six-dimensional chiral theory) the global symmetry group and its compact part are larger. Again this also holds for the 'exceptional' supergravities. Hence for $\mathcal{N}>2$ one always has $H_{\mathrm{R}} \subseteq H$.

For $\mathcal{N}=2$, however, this is not always the case. In particular, in the absence of hyper multiplets there is an $S U(2) \subseteq H_{\mathrm{R}}$ part missing in $H$ for all dimensions. For example, pure $\mathcal{N}=2$ supergravity in $D=5$ should have $S U(2)$ R-symmetry but there are no scalars giving a scalar manifold $G / H$ with $S U(2) \subseteq H$. For precisely such cases there is also a problem with the correspondence between the Kac-Moody algebra and supergravity, as the former does not contain all the potential gaugings of the latter. In particular, the potentials corresponding to the gaugings of $S U(2) \subseteq H_{\mathrm{R}}$ in $D \leq 5$ are not present in the Kac-Moody algebra. This mismatch has been noted in [36]. In section 3 a resolution is proposed by including an additional 'empty' $S U(2) / S U(2)$ scalar manifold, such that $H_{\mathrm{R}} \subseteq H$ holds for these cases as well 6 Of course, the additional compact factor does not

\footnotetext{
${ }^{5}$ In dimensions lower than $D=3$ the R-symmetry group $H_{\mathrm{R}}$ of maximal supergravity is again identical to $H$, though $H$ is now infinite-dimensional [32-35].

${ }^{6}$ Such a factor appears in [37] but apparently has been replaced by "1" in the subsequent literature.
} 
introduce any physical degrees of freedom. However, we will see that the corresponding extended semi-simple algebra does contain the possible gaugings of this compact factor, and agrees perfectly with the results of [36] 7

Summarising, the purpose of this paper is twofold:

- Firstly, we make a proposal for the extensions for semi-simple $\mathfrak{g}$, i.e. the analogon of the affine, over- and very-extension of simple $\mathfrak{g}$. The corresponding extensions will turn out to be quotients of certain derived Kac-Moody algebras. We will present a number of arguments why these extensions are the relevant ones in the context of supergravity.

- Secondly, we argue that the problematic case with $H_{\mathrm{R}} \nsubseteq H$ can be remedied by the extension of the scalar coset with the missing compact factor. For $\mathcal{N}=2$ supergravity without hyper multiplets this is an $S U(2) / S U(2)$ factor. Also in the absence of hyper multiplets one then has $H_{\mathrm{R}} \subseteq H$ and $\mathfrak{g}$ semi-simple in three dimensions. (In the presence of hyper multiplets $H_{\mathrm{R}} \subseteq H$ follows directly from the scalar manifold of the hyper multiplets.)

The outline of this paper is as follows. In section 2 we will present a proposal for the extensions of semi-simple Lie algebras. In section 3 these will be applied to a pair of $\mathcal{N}=2$ supergravity examples: one with and one without hyper multiplets. In section 4 the relation of these examples to $\mathfrak{e}_{11}$ is discussed. Our conclusions are presented in section 5. In appendix $\mathrm{A}$ we review the supersymmetry algebra of pure $\mathcal{N}=2$ supergravity in $D=5$ and show the possibility to include certain five-forms. Some general remarks and a particular example of $\mathcal{N}=1$ supergravity are discussed in appendix B. Finally, appendix C] contains the decomposition tables of the Kac-Moody algebras corresponding to the supergravity examples.

\section{Extensions of semi-simple Lie algebras}

In this section we discuss the general problem of obtaining Kac-Moody extensions of direct sums of finite-dimensional simple Lie algebras.

\subsection{Review of the extension process for simple Lie algebras}

For a complex, finite-dimensional and simple Lie algebra $\mathfrak{g}$ there exists a standard process of extending the Dynkin diagram by three nodes to obtain the so-called very-extension $\mathfrak{g}^{+++}[27,28]$. This extension process consists of three steps, where the first additional node leads to the so-called non-twisted affine extension which we denote here as $\mathfrak{g}^{+}$. The way the affine node is attached to the Dynkin diagram of $\mathfrak{g}$ is governed by the highest root of $\mathfrak{g}$. A list of the diagrams of all non-twisted $\mathfrak{g}^{+}$can be found for example in [39]. As a vector space $\mathfrak{g}^{+}$is isomorphic to $\mathfrak{g}\left[\left[t, t^{-1}\right]\right] \oplus \mathbb{C} c \oplus \mathbb{C} d$, i.e. the centrally extended loop algebra over $\mathfrak{g}$ with spectral parameter $t$, central element $c$ and derivation $-t \frac{d}{d t}$. From the

\footnotetext{
${ }^{7}$ Similarly, the absence of four-forms in $\mathfrak{g}_{2}^{++}$led to a paradox concerning higher-order corrections to this five-dimensional supergravity [38]. We expect our proposal to resolve this puzzle as well.
} 
algebraic point of view the derivation $d$ serves to desingularize the inner product $(\cdot \mid \cdot)$ on the Cartan subalgebra. Since $c$ is central it satisfies $(c \mid h)=0$ for all Cartan generators $h$ of the finite-dimensional $\mathfrak{g}$ and also $(c \mid c)=0$. By introducing the derivation $d$ with $(d \mid c)=-1$ this degeneracy of the inner product is alleviated 8 If $\mathfrak{g}$ is of rank $r$, so that there are $r$ independent Cartan generators in $\mathfrak{g}$, the affine extension $\mathfrak{g}^{+}$has $r+2$ commuting diagonal elements, exceeding the number of nodes of the Dynkin diagram by one. We will always use the notation $\mathfrak{g}^{+}$in this paper to refer to the Kac-Moody algebra $\mathfrak{g}\left[\left[t, t^{-1}\right]\right] \oplus \mathbb{C} c \oplus \mathbb{C} d$ and call it the affine version of the simple Lie algebra $\mathfrak{g}$.

The next step in the extension process leads to the over-extension $\mathfrak{g}^{++}$and can be thought of as giving a Dynkin-diagrammatic home to the derivation $d$ so that the number of nodes agrees again with the number of independent diagonal elements. In order to ensure all the properties of $d$ on the affine subalgebra $\mathfrak{g}^{+}$the new node has to be joined with a single undirected line to the affine node. The resulting algebra has inner product of Lorentzian signature on the Cartan subalgebra.9 In the last step (very-extension) one adjoins another node with a single line to the hyperbolic node to obtain the Lorentzian algebra $\mathfrak{g}^{+++}[28]$. As the number of Cartan subalgebra elements will be of importance for our proposal for extending semi-simple algebras we summarize this again: For simple $\mathfrak{g}$ of rank $r$ there are $r+2, r+2$ and $r+3$ independent Cartan subalgebra generators for $\mathfrak{g}^{+}, \mathfrak{g}^{++}$and $\mathfrak{g}^{+++}$, respectively. In contrast, the loop algebra $\mathfrak{g}\left[\left[t, t^{-1}\right]\right]$ has $r$ Cartan subalgebra elements.

The affine algebra $\mathfrak{g}^{+}$arises from gravity models for simple $\mathfrak{g}$ as follows. We consider gravity in $D=3$ coupled to a $G / H$ scalar coset, where we now assume that a real form of $\mathfrak{g}$ has been chosen which is the Lie algebra of $G$. As always, $H=K(G)$ is the maximal compact subgroup of $G$ and describes the local symmetries. In the reduction to $D=2$ the affine extension $\mathfrak{g}^{+}$arises as the new and larger symmetry algebra as can be shown by considering a linear system based on $\mathfrak{g}^{+}[5,43]$. This symmetry is commonly referred to as Geroch symmetry [44]; the importance of the central extension and derivation were first noticed in $[45,46]$. The central element is related to the size of the non-compact twodimensional space-time, whereas the derivation $d$ is related to the size of the circle used in the reduction from $D=3$ to $D=210$ The spectral parameter $t$ is needed to distinguish and organise the infinity of independent auxiliary scalar fields dual to the reduced scalars one can introduce in $D=2$ (the so-called dual potentials $[5,43]$ ).

\subsection{Extending semi-simple Lie algebras}

If one instead starts with a direct sum, say $\mathfrak{g}_{a} \oplus \mathfrak{g}_{b}$ of simple finite-dimensional Lie algebras rather than a single simple Lie algebra, the extension process described above is not uniquely defined any longer. The ambiguity arises already for the affine extension when defining $\left(\mathfrak{g}_{a} \oplus \mathfrak{g}_{b}\right)^{+}$. One possibility would be to define $\left(\mathfrak{g}_{a} \oplus \mathfrak{g}_{b}\right)^{+}=\mathfrak{g}_{a}^{+} \oplus \mathfrak{g}_{b}^{+}$, treating the

\footnotetext{
${ }^{8}$ The elements $c$ and $d$ can be thought of as two independent light-cone coordinates.

${ }^{9}$ In many physically interesting cases the associated Kac-Moody algebra is hyperbolic such that the BKL limit near a space-like singularity will exhibit chaos [40-42].

${ }^{10}$ More precisely, in conformal gauge the $D=2$ metric has as its one independent component the conformal factor which is acted upon by symmetry transformations in the $c$ direction. If the $G / H$ coset arises from the reduction of some higher dimensional model $d$ acts on the overall size of all compact direction.
} 


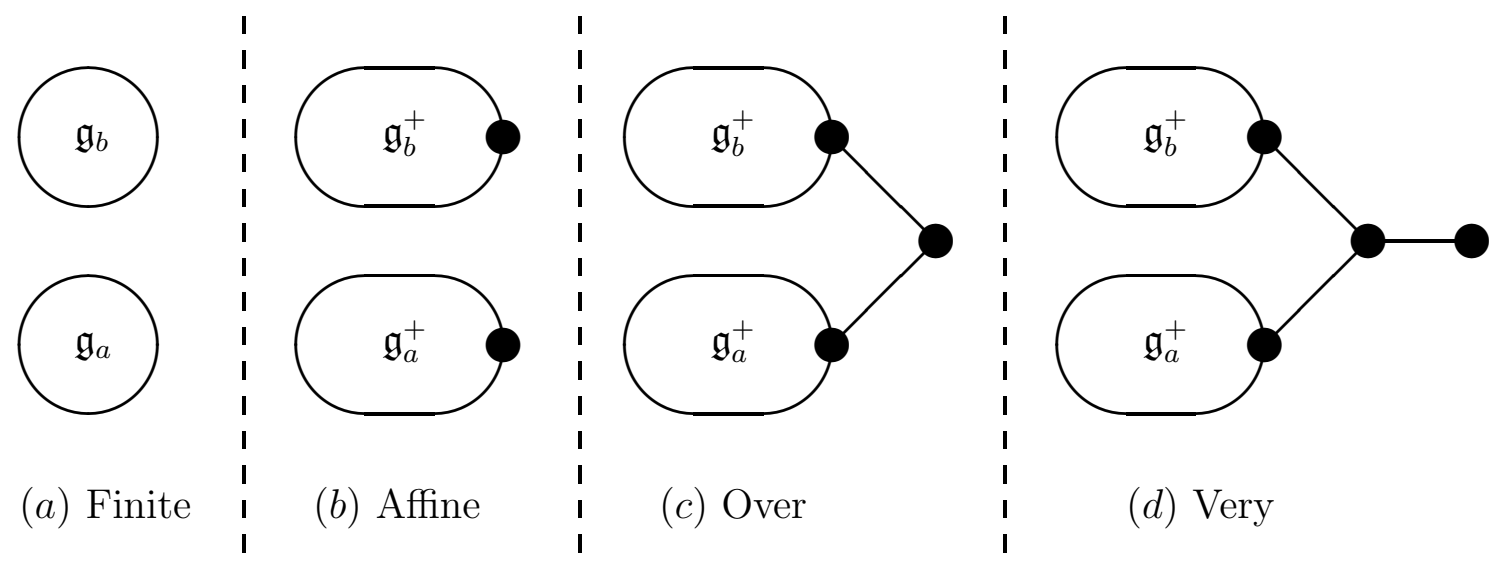

Figure 1: The extension process for semi-simple $\mathfrak{g}_{a} \oplus \mathfrak{g}_{b}$ in terms of Dynkin diagrams. The marked nodes on the blobs for the affine algebras correspond to the affine nodes of the non-twisted affine extensions of the simple $\mathfrak{g}_{a}$ and $\mathfrak{g}_{b}$. As explained in the text the simple algebras $\left(\mathfrak{g}_{a} \oplus \mathfrak{g}_{b}\right)^{++}$and $\left(\mathfrak{g}_{a} \oplus \mathfrak{g}_{b}\right)^{+++}$are obtained from these Dynkin diagrams by taking the quotient of the derived algebra by its center. This amounts to removing the derivation and central element present in the Kac-Moody algebras described by these Dynkin diagrams. The affine algebra $\left(\mathfrak{g}_{a} \oplus \mathfrak{g}_{b}\right)^{++}$is defined as in (31).

two algebras also completely independently in the extension process. This results in two independent central elements and two independent derivations for the two summands. However, the loop algebra construction also suggests another possibility, namely to consider $\left(\mathfrak{g}_{a} \oplus \mathfrak{g}_{b}\right)^{+}=\left(\mathfrak{g}_{a} \oplus \mathfrak{g}_{b}\right)\left[\left[t, t^{-1}\right]\right] \oplus \mathbb{C} c \oplus \mathbb{C} d$. In this case there is a common spectral parameter, a single common derivation $d=-t \frac{d}{d t}$ and a common central element. We note that this definition does not give a Kac-Moody algebra; it is closely related to the Kac-Moody algebra $\mathfrak{g}_{a}^{+} \oplus \mathfrak{g}_{b}^{+}$from which it differs by having the two central charges $c_{a}$ and $c_{b}$ as well as the two derivations $d_{a}$ and $d_{b}$ identified.

From the perspective of the connection to (super-)gravity theories the second (non Kac-Moody) option is preferred when one repeats the arguments leading to the emergence of the affine symmetry reviewed above. Even if $\mathfrak{g}$ is not simple the reduction to $D=2$ should still give rise to only one central element and one derivation since they have a geometric origin. Therefore the symmetry consideration of gravity coupled to scalar cosets leads to the second option of defining the affine extension of a semi-simple $\mathfrak{g}_{a} \oplus \mathfrak{g}_{b}$.

For this reason we will adopt from now on the definition

$$
\left(\mathfrak{g}_{a} \oplus \mathfrak{g}_{b}\right)^{+}:=\left(\mathfrak{g}_{a} \oplus \mathfrak{g}_{b}\right)\left[\left[t, t^{-1}\right]\right] \oplus \mathbb{C} c \oplus \mathbb{C} d
$$

Of course, in terms of loop algebras one has $\left(\mathfrak{g}_{a} \oplus \mathfrak{g}_{b}\right)\left[\left[t, t^{-1}\right]\right]=\mathfrak{g}_{a}\left[\left[t, t^{-1}\right]\right] \oplus \mathfrak{g}_{b}\left[\left[t, t^{-1}\right]\right]$. As we have stressed, equation (3) does not correspond to a Kac-Moody algebra but is related to the Kac-Moody algebra $\mathfrak{g}_{a}^{+} \oplus \mathfrak{g}_{b}^{+}$, the Dynkin diagram of which is displayed in fig. 1(b), by identifying the two central elements with each other and by identifying also the two derivations.

Continuing to the over-extension $\left(\mathfrak{g}_{a} \oplus \mathfrak{g}_{b}\right)^{++}$it is natural to construct a Kac-Moody algebra where the single derivation $d$ of (3) is included naturally as for the simple case. 
Since the derivation acts on both loop algebras $\mathfrak{g}_{a}\left[\left[t, t^{-1}\right]\right]$ and $\mathfrak{g}_{b}\left[\left[t, t^{-1}\right]\right]$ alike, the two disjoint Dynkin diagrams should be joined via the hyperbolic node, see fig. 1(c). The Cartan element of this common hyperbolic node will act as the derivation on both loop algebras if the link is a single line and undirected. The generalised Cartan matrix encoded by the diagram of fig. 1(c) has one zero eigenvalue which corresponds to the diagonal generator $\tilde{c}=c_{a}-c_{b}$ in terms of the central elements $c_{a}$ and $c_{b}$ of the constituent $\mathfrak{g}_{a}^{+}$and $\mathfrak{g}_{b}^{+}$. The Kac-Moody algebra $\tilde{\mathfrak{g}}^{++}$defined by fig. 1(c) requires therefore a new derivation $\tilde{d}$ to desingularize the inner product which is degenerate due to the presence of $\tilde{c}$. Furthermore, the derived algebra $\left(\tilde{\mathfrak{g}}^{++}\right)^{\prime}=\left[\tilde{\mathfrak{g}}^{++}, \tilde{\mathfrak{g}}^{++}\right]$of the Kac-Moody algebra $\tilde{\mathfrak{g}}^{++}$is isomorphic to $\tilde{\mathfrak{g}}^{++}$without $\tilde{d}$. The generator $\tilde{c}=c_{a}-c_{b}$ is central in $\tilde{\mathfrak{g}}^{++}$. Taking the quotient of the derived algebra by the center spanned by $\tilde{c}$ will lead to a simple, infinite-dimensional Liealgebra [39] which we will call the over-extension $\left(\mathfrak{g}_{a} \oplus \mathfrak{g}_{b}\right)^{++}$of the semi-simple algebra $\mathfrak{g}_{a} \oplus \mathfrak{g}_{b}$ :

$$
\left(\mathfrak{g}_{a} \oplus \mathfrak{g}_{b}\right)^{++}:=\left(\tilde{\mathfrak{g}}^{++}\right)^{\prime} /\langle\tilde{c}\rangle .
$$

It is not a Kac-Moody algebra but differs from one, i.e. $\tilde{\mathfrak{g}}^{++}$, only in the diagonal elements in the same way that a loop algebra $\mathfrak{g}\left[\left[t, t^{-1}\right]\right]$ differs from the affine algebra $\mathfrak{g}^{+}$. Moreover, the quotient by the one-dimensional space spanned by $\tilde{c}=c_{a}-c_{b}$ identifies the two individual central elements of the affine algebras $\mathfrak{g}_{a}^{+}$and $\mathfrak{g}_{b}^{+}$which is exactly what we argued for on the basis of the Geroch symmetry in $D=2$ gravity.

From the construction of $\left(\mathfrak{g}_{a} \oplus \mathfrak{g}_{b}\right)^{++}$it is now easy to construct the very-extension $\left(\mathfrak{g}_{a} \oplus \mathfrak{g}_{b}\right)^{+++}$by adding another node to the Dynkin diagram. It is again understood that $\left(\mathfrak{g}_{a} \oplus \mathfrak{g}_{b}\right)^{+++}$is constructed from the corresponding Kac-Moody algebra $\tilde{\mathfrak{g}}^{+++}$, with Dynkin diagram shown in fig. 1(d), by removing the derivation and central element in the same way as described above 11 By abuse of notation we will sometimes refer to the Dynkin diagram of $\tilde{\mathfrak{g}}^{+++}$as the Dynkin diagram of $\left(\mathfrak{g}_{a} \oplus \mathfrak{g}_{b}\right)^{+++}$.

There is a natural extension of our proposal to the case $\mathfrak{g}_{a} \oplus \mathfrak{g}_{b} \oplus \mathfrak{g}_{c} \oplus \ldots$, consisting of $n$ simple factors. On general grounds there will now be $n-1$ central elements $\tilde{c}_{a}=c_{a}-c_{b}$, $\tilde{c}_{b}=c_{b}-c_{c}, \ldots$ such that again in $\left(\mathfrak{g}_{a} \oplus \mathfrak{g}_{b} \oplus \mathfrak{g}_{c} \oplus \ldots\right)^{++}$all originally distinct central charges are identified in agreement with the Geroch symmetry. This extends also to the very-extended case.

The very-extended algebras $\left(\mathfrak{g}_{a} \oplus \mathfrak{g}_{b}\right)^{+++}$we construct in the fashion described above have $r_{a}+r_{b}+3$ diagonal elements for finite-dimensional $\mathfrak{g}_{a}$ and $\mathfrak{g}_{b}$ of rank $r_{a}$ and $r_{b}$, respectively. The signature of the inner product on these diagonal elements is $\left(r_{a}+r_{b}+2,1\right)$ and therefore of Lorentzian type 12

We note that one can also use different real forms of $\mathfrak{g}_{a}$ and $\mathfrak{g}_{b}$ in this extension process, they do not need to be in split real form. However, we demand from the symmetries of the reduction of $D=3$ gravity that the affine, over- and very-extended nodes be noncompact. Of course, the resulting diagram then should be an allowed almost split real form of the very-extended Kac-Moody algebra, see e.g. [47,48]. The properly defined Weyl groups of the quotient Lie algebras introduced in this section should be related to U-duality in low dimensions [49].

\footnotetext{
${ }^{11}$ It can be seen that $c_{a}-c_{b}$ is still central in $\tilde{\mathfrak{g}}^{+++}$and that the center is one-dimensional.

${ }^{12}$ This follows since there is an $\left(r_{a}+r_{b}+2\right)$-dimensional space-like subspace, a one-dimensional kernel and at least a one-dimensional time-like subspace. A dimension count then shows that the quotient by the kernel has signature $\left(r_{a}+r_{b}+2,1\right)$.
} 


\section{Applications to $\mathcal{N}=2$ supergravity}

In this section we will discuss the emergence of semi-simple Lie algebras in $\mathcal{N}=2$ supergravity, and analyse in detail a pair of examples of very-extended semi-simple Lie algebras. These illustrate our proposal of the previous section and in addition will provide a number of consistency checks.

\subsection{General aspects}

As discussed in the introduction, the $\mathcal{N}=2$ theories have the highest number of supercharges that allow for inhomogenous scalar manifolds. Of course $\mathcal{N}=2$ supersymmetry does impose a number of geometric restrictions on these spaces. Most importantly, the scalar manifolds split up in two parts, parametrised by scalars of the vector 13 and hyper multiplets, respectively:

$$
\mathcal{M}=\mathcal{M}_{V} \times \mathcal{M}_{H}
$$

Further requirements on the scalar manifold $\mathcal{M}_{V}$ are dimension-dependent: it is very special real, special Kähler and quaternionic-Kähler in $D=5,4,3$, respectively. In $D=6$ it is given by a particular homogeneous space. The scalar manifold $\mathcal{M}_{H}$ is quaternionicKähler in any dimension $3 \leq D \leq 6$. See e.g. [29] and references therein for further details on these spaces.

We will be concerned with the subset of $\mathcal{N}=2$ supergravities whose scalar manifolds are homogeneous spaces. These have been classified in $[50,51]$. In particular we are interested in the global symmetries of these theories. From the split of scalar manifolds (5) it follows that the symmetries will generically be semi-simple. Only in the absence of hyper multiplets 14 can one have a simple $G$.

Furthermore, the R-symmetry is not always contained in the compact subgroups of $G$ for $\mathcal{N}=2$ supergravities. This is easiest to see in three dimensions where one has only vector and hyper multiplets. Both multiplets consist of the same fields, being four scalars and two dilatini. They only differ in the way they transform under the R-symmetry, which is $H_{\mathrm{R}}=S O(4) \simeq S U(2) \times S U(2)$ : only one $S U(2)$ factor acts on the scalars of the vector multiplets, while the other factor only acts on the scalars of the hyper multiplets [25]. This implies that both factors of $H_{\mathrm{R}}$ will be contained in $H$ if and only if hyper multiplets are present as well. The same will hold for the possible uplift of these theories to higher dimensions.

Although the reasoning is completely general and will apply to all $\mathcal{N}=2$ theories, it may be instructive to consider specific examples of both kinds (i.e. with and without hyper multiplets):

\footnotetext{
${ }^{13}$ The vector multiplet does not comprise any scalars in six dimensions, but instead there is a tensor multiplet which does. For simplicity we will refer to the corresponding scalar manifold as $\mathcal{M}_{V}$ as well.

${ }^{14}$ We will not consider the case of only hyper multiplets as these theories only live in $D=3$.

${ }^{15}$ The Kac-Moody extensions of simple algebras associated to $\mathcal{N}=2$ theories without hyper multiplets have been discussed in $[16,17]$.
} 
- Pure $\mathcal{N}=2$ supergravity in five dimensions consists of only the graviton multiplet. Its bosonic sector comprises the graviton and a vector, while its fermions are a pair of symplectic Majorana gravitini.

- One can couple this theory to seven hyper multiplets whose scalars parametrise an $F_{4} /(S U(2) \times S p(3))$ scalar coset.

These will be referred to as the pure theory and the coupled theory, respectively. Interestingly, both can be obtained as a truncation of $\mathcal{N}=8$ supergravity $[26,37,52]$. In table 2 the global symmetry group and its compact subgroup are given for these theories and their dimensional reductions. In addition we indicate the R-symmetry group.

\begin{tabular}{||c||c||c|c||c|c||}
\hline$D$ & $H_{\mathrm{R}}$ & $G_{\text {pure }}$ & $H_{\text {pure }}$ & $G_{\text {coupled }}$ & $H_{\text {coupled }}$ \\
\hline \hline 5 & $S U(2)$ & 1 & 1 & $F_{4}$ & $S U(2) \times S p(3)$ \\
\hline 4 & $U(2)$ & $S L(2)$ & $S O(2)$ & $S L(2) \times F_{4}$ & $U(2) \times S p(3)$ \\
\hline 3 & $S O(4)$ & $G_{2}$ & $S O(4)$ & $G_{2} \times F_{4}$ & $S U(2) \times S O(4) \times S p(3)$ \\
\hline
\end{tabular}

Table 2: The global symmetries $G$ and their compact subgroups $H$ of two five-dimensional $\mathcal{N}=2$ supergravities and their dimensional reductions. The first is the pure theory while the second is coupled to seven hyper multiplets.

In line with the discussion above, the R-symmetry group is not contained in $H_{\text {pure }}$. This is easy to see in five and four dimensions. In three dimensions one might think that they coincide. However, the pure theory reduces to two vector multiplets (and a gravity multiplet) in three dimensions. Only one of the $S U(2)$ factors of $H_{\mathrm{R}}$ acts on the scalars contained in the vectors. In contrast, $H_{\text {pure }}=S O(4)$ acts on all scalars of the theory. Consequently $H$ and $H_{\mathrm{R}}$ should not be identified; only an $S U(2)$ factor of both groups coincide. Similarly, in four dimensions only an $S O(2)$ factor coincides. Therefore the pure theory always has an $S U(2)$ factor of $H_{\mathrm{R}}$ missing in all dimensions, while it has a simple symmetry $G_{2}$ in three dimensions. In contrast, the theory coupled to hyper multiplets does have $H_{\mathrm{R}} \subseteq H_{\text {coupled }}$, but has the semi-simple symmetry $G_{2} \times F_{4}$ in three dimensions.

We will first discuss the extended semi-simple Lie algebra associated with the coupled theory before addressing the pure theory.

\subsection{Very-extended $\mathfrak{g}_{2} \oplus \mathfrak{f}_{4}$ and the coupled theory}

The Dynkin diagram of $\left(\mathfrak{g}_{2} \oplus \mathfrak{f}_{4}\right)^{+++}$according to our general construction of section 2 can be found in figure 2 ${ }^{16}$ A regular $\mathfrak{s l}(5)$ subalgebra has been indicated as well, whose indices will be interpreted as space-time indices 17 The decomposition into generators of this $\mathfrak{s l}(5)$ with up to five space-time indices can be found in table 5 in appendix $\mathbb{C}$. These correspond to the following physical degrees of freedom (at level $\left(l_{1}, l_{2}\right)$ ):

\footnotetext{
${ }^{16}$ Recall that $\left(\mathfrak{g}_{2} \oplus \mathfrak{f}_{4}\right)^{+++}$strictly speaking does not admit a Dynkin diagram, but we refer to the diagram of the underlying Kac-Moody algebra as its Dynkin diagram.

${ }^{17}$ For more details and examples of Kac-Moody decompositions see e.g. [16].
} 
- $(0,0)$ : the traceless part of the metric, carrying 24 fields of which 10 will be eliminated due to the local Lorentz symmetry,

- $(0,0)$ : a scalar field which provides the trace of the metric,

- $(0,0)$ : scalars in the adjoint of $\mathfrak{f}_{4}$, subject to the local $S U(2) \times S p(3)$ symmetry,

- $(1,0)$ : a vector,

- $(2,0)$ : a two-form, which is interpreted as dual to the vector,

- $(3,0)$ : a generator with mixed symmetry properties, which is interpreted as the dual graviton,

- $(0,1)$ : three-forms in the adjoint of $\mathfrak{f}_{4}$, which are interpreted as dual to the scalars.

The metric, vector and their duals belong to the graviton multiplet.The remaining degrees of freedom are spanned by the scalars of the seven hyper multiplets and their duals. Note that one finds 52 scalars transforming in the adjoint representation of $\mathfrak{f}_{4}$, while there are only 28 physical scalars in the theory. However, one has to divide out by the compact subgroup of the internal symmetry group $F_{4}$, which is in this case $S U(2) \times S p(3)$ and therefore eliminates 24 scalars. Similarly, one finds 52 three-forms which can be seen as the duals to the scalars. We expect that supersymmetry will impose 24 linear constraints on the field strengths of these two-forms. This reduces the number of independent threeforms to 28 , which will be related to the physical scalars via a Hodge duality relation. This is completely analogous to the way the $S L(2) / S O(2)$ sector of IIB supergravity appears in $E_{11}$ (see e.g. [16] and also [21,53]). In that case the compact $S O(2)$ subgroup eliminates one of the three scalars. In addition there is a single linear constraint on the field strengths of the dual eight-forms and duality relations between the remaining scalars and eight-forms $[21,53]$.

Since a crucial aspect of our proposal is that the relevant symmetry is the quotient of the derived algebra of the Kac-Moody algebra given by the diagram 2 we examine the effect of the quotient in this case in detail. The $(10 \times 10)$ Cartan matrix encoded in figure 2 has one zero eigenvalue so that the associated Kac-Moody algebra must have eleven independent Cartan generators, including one central charge $\tilde{c}$ and the associated derivation $\tilde{d}$. The central charge $\tilde{c}$ is the difference of the central charges of the affine $\mathfrak{f}_{4}^{+}$and $\mathfrak{g}_{2}^{+}$diagrams contained in fig. 2, The transition to the derived algebra removes $\tilde{d}$ and the quotient eliminates $\tilde{c}$ so that the resulting algebra has only nine commuting diagonalisable elements. This has to be compared to the number of diagonal metric components and dilaton-like scalars in the scalar coset. In $D=5$ there are five diagonal metric components and for the $F_{4}$ coset there are four dilaton-like scalars so that the numbers agree precisely.

With this understanding, indeed the above decomposition coincides exactly with the physical degrees of freedom (and their duals) of this theory. The other generators do not correspond to any (known) propagating degrees of freedom. Nevertheless, a subset of these is known to play an interesting role in supergravity. These are the purely antisymmetric $(D-1)$ - and $D$-forms, as discussed in the introduction. In the case at hand these are 


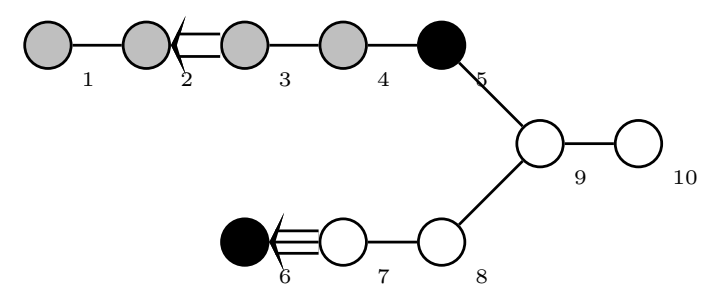

Figure 2: $\left(\mathfrak{g}_{2} \oplus \mathfrak{f}_{4}\right)^{+++}$decomposed as $\mathfrak{s l}(5) \oplus \mathfrak{f}_{4}$, corresponding to a $D=5$ theory with an $F_{4}$ internal symmetry group. The colouring in all our diagrams is such that white nodes correspond to the non-compact gravity line whereas the grey nodes indicate the internal symmetry group. The black nodes are the ones with respect to which the level decomposition is performed.

- $(1,1)$ : four-forms in the adjoint of $\mathfrak{f}_{4}$,

- $(2,1)$ : five-forms in the adjoint of $\mathfrak{f}_{4}$.

The four-forms are interpreted as dual to the gauge parameters $[18,19]$. These specify the possible embeddings of a one-dimensional gauge group in $F_{4}$. In addition the veryextended algebra predicts the possibility to include an $\mathfrak{f}_{4}$ adjoint of space-time filling five-forms, whose role is yet to be understood.

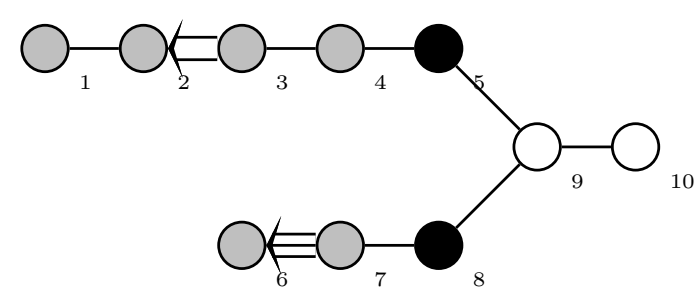

Figure 3: $\left(\mathfrak{g}_{2} \oplus \mathfrak{f}_{4}\right)^{+++}$decomposed as $\mathfrak{s l}(3) \oplus \mathfrak{g}_{2} \oplus \mathfrak{f}_{4}$, corresponding to a $D=3$ theory with a $G_{2} \times F_{4}$ internal symmetry group.

In addition to the previous five-dimensional perspective, we would also like to consider the decomposition of very-extended $\mathfrak{g}_{2} \oplus \mathfrak{f}_{4}$ with respect to its $\mathfrak{s l}(3)$ subalgebra, corresponding to the theory reduced to three dimensions. The associated Dynkin diagram is given in figure 3 while the result of the decomposition can be found in table 6. It can easily be seen that at levels $\left(l_{1}, l_{2}\right)=(0,0),(0,1)$ and $(1,0)$ one finds exactly the generators that are associated to the physical degrees of freedom of the reduced supergravity theory. These are the graviton and its trace, and scalars in the adjoints of $\mathfrak{g}_{2}$ and $\mathfrak{f}_{4}$ and their dual vectors. In addition the very-extended algebra contains the following non-propagating degrees of freedom:

- two-forms in the $\left(\mathfrak{g}_{2}, \mathfrak{f}_{4}\right)$ representations $(\mathbf{1} \oplus \mathbf{2 7}, \mathbf{1}) \oplus(\mathbf{1}, \mathbf{1} \oplus \mathbf{3 2 4}) \oplus(\mathbf{1 4}, \mathbf{5 2})$,

- three-forms in the $\left(\mathfrak{g}_{2}, \mathfrak{f}_{4}\right)$ representations $(\mathbf{1} \oplus \mathbf{1 4} \oplus \mathbf{2 7} \oplus \mathbf{7 7}, \mathbf{5 2}) \oplus(\mathbf{1 4}, \mathbf{1} \oplus \mathbf{5 2} \oplus$ $324 \oplus 1274)$. 
The two-forms are in one-to-one correspondence with the components of the embedding tensor that parametrise the most general gaugings of this theory [54]. Therefore the above features are exactly what one would expect for the algebra corresponding to this $D=3$ theory.

Note that in the above we have interpreted the lower branch of the Dynkin diagram 2 as corresponding to the scalar manifold of the vectors, while the upper branch corresponds to the hyper manifolds. In other words, we have chosen to identify

$$
\mathcal{M}_{V}=\frac{G_{2}}{S O(4)}, \quad \mathcal{M}_{H}=\frac{F_{4}}{S U(2) \times S p(3)},
$$

in three dimensions. With these identifications one can only uplift to the coupled theories in table 2. In this uplift the scalar manifold $F_{4} /(S U(2) \times S p(3))$ is unaffected, while the scalar manifold $G_{2} / S O(4)$ is deconstructed to yield gravity and vectors in the higher dimensions.

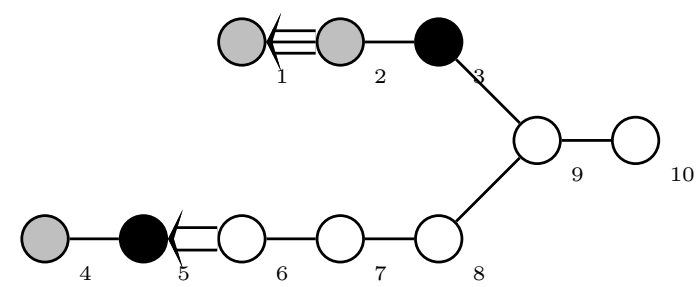

Figure 4: $\left(\mathfrak{g}_{2} \oplus \mathfrak{f}_{4}\right)^{+++}$decomposed as $\mathfrak{s l}(6) \oplus \mathfrak{g}_{2} \oplus \mathfrak{s l}(2)$, corresponding to a $D=6$ theory with a $G_{2} \times S L(2)$ internal symmetry group.

However, in three dimensions the vector and hyper multiplets are interchangable, and hence one could have made identification (6) with $\mathcal{M}_{V}$ and $\mathcal{M}_{H}$ interchanged. In this case the scalar manifold $G_{2} / S O(4)$ is unaffected by the uplift, while the other factor $F_{4} /(S U(2) \times S p(3))$ is deconstructed in different higher-dimensional fields. With such identifications the higher-dimensional origin is therefore completely different. In particular, the highest dimension to which this theory can be uplifted is six, corresponding to the $\mathfrak{s l}(6)$ regular subalgebra of very-extended $\mathfrak{g}_{2} \oplus \mathfrak{f}_{4}$ shown in figure 4 . This corresponds to the six-dimensional chiral $\mathcal{N}=2$ supergravity that reduces to the $F_{4} /(S U(2) \times S p(3))$ coset in $D=3$ [55], coupled to two hyper multiplets parametrising a $G_{2} / S O(4)$ scalar manifold. We have checked that the decomposition of very-extended $\mathfrak{g}_{2} \oplus \mathfrak{f}_{4}$ with respect to this $\mathfrak{s l}(6)$ regular subalgebra, given in table 7 , gives rise to the correct physical degrees of freedom. In addition it includes the following non-propagating degrees of freedom:

- five-forms in the $\left(\mathfrak{s l}(2), \mathfrak{g}_{2}\right)$ representations $(\mathbf{2} \oplus \mathbf{4}, \mathbf{1}) \oplus(\mathbf{2}, \mathbf{1 4})$,

- $\operatorname{six}$-forms in the $\left(\mathfrak{s l}(2), \mathfrak{g}_{2}\right)$ representations $(\mathbf{3} \oplus \mathbf{3} \oplus \mathbf{5}, \mathbf{1}) \oplus(\mathbf{1} \oplus \mathbf{3}, \mathbf{1 4})$.

The former should parametrise the possible gaugings of the $S L(2) \times G_{2}$ internal symmetry with the $S L(2)$ doublet of vectors. 


\subsection{Very-extended $\mathfrak{g}_{2} \oplus \mathfrak{s u}(2)$ and the pure theory}

We will now discuss the pure $\mathcal{N}=2, D=5$ theory introduced earlier, i.e. the case without hyper multiplets, and the corresponding very-extended algebra.

First let us address in more detail why the case without hyper multiplets, where the R-symmetry is not contained in $H$, leads to a mismatch from the Kac-Moody point of view. We will do this in the context of the $D=5$ pure theory, whose physical bosonic degrees of freedom reduce in three dimensions to two vector multiplets parametrising the coset

$$
\mathcal{M}_{V}=\frac{G_{2}}{S O(4)} .
$$

The corresponding Kac-Moody algebra is $\mathfrak{g}_{2}^{+++}$, whose Dynkin diagram with a regular $\mathfrak{s l}(5)$ subalgebra indicated is given in figure 5 . The corresponding decomposition is given in table 8 in appendix C] see also [16]. At lowest levels $l$ we find the expected physical degrees of freedom:

- 0: the traceless part of the metric, carrying 24 degrees of freedom of which 10 will be eliminated due to the local Lorentz symmetry,

- 0: a scalar which provides the trace of the metric,

- 1: a vector,

- 2: a two-form, which is interpreted as dual to the vector,

- 3: a generator with mixed symmetry properties, interpreted as the dual graviton.

The other generators all have mixed symmetries and do not correspond to propagating degrees of freedom.

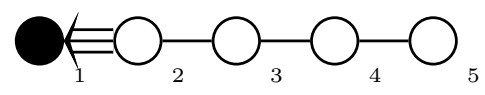

Figure 5: $\mathfrak{g}_{2}^{+++}$decomposed as $\mathfrak{s l}(5)$, corresponding to a $D=5$ theory.

For this particular theory, however, it has been explicitly calculated which gauge potentials can be included, i.e. on which gauge potentials the supersymmetry algebra can be realised [36]. In addition to the vector and its dual two-form present in $\mathfrak{g}_{2}^{+++}$, threeand four-forms transforming under the R-symmetry $S U(2)$ were found. The four-forms should be expected: they can be seen as the potentials dual to the gauging parameters. Indeed, in the pure theory one can gauge a $U(1)$ group [56], whose embedding in $S U(2)$ is described by a triplet of parameters. However, since the original bosonic fields of the theory (i.e. the metric, the vector and their duals) are invariant under the R-symmetry the corresponding Kac-Moody algebra will not contain $H_{\mathrm{R}}$ and hence will miss the potentials corresponding to its gauging [57]. 
A clue for the resolution of this puzzle comes from the triplet of three-forms. In $D=5$ these would in general be interpreted as dual to scalars, which the theory does not have and hence would introduce additional propagating degrees of freedom. However, it was found that, in order to realise supersymmetry on them, their field strengths necessarily vanish, and hence they correspond to non-propagating degrees of freedom. This is similar to the $S L(2) / S O(2)$ coset of IIB supergravity (or the $F_{4} /(S U(2) \times S p(3))$ coset of the coupled theory considered in section 3.2). In the IIB case there was one linear constraint on the field strengths of the $(D-2)$-forms, corresponding to the local $S O(2)$ symmetry reducing the number of scalars from three to two. Therefore a natural interpretation for the three-forms in $D=5$ is as dual to an $S U(2) / S U(2)$ coset. As this is a compact factor there are no scalars associated to it, and the dual $(D-2)$-forms are subject to three linear constraints on their field strengths, which therefore vanish. Hence the results of [36] indicate that one should include an $S U(2) / S U(2)$ scalar manifold in the $D=5$ pure theory (see also [37]). This does not mix with the other fields under dimensional reduction and the resulting coset in $D=3$ is

$$
\mathcal{M}_{V}=\frac{G_{2}}{S O(4)}, \quad \mathcal{M}_{H}=\frac{S U(2)}{S U(2)},
$$

and thus corresponds to a global semi-simple algebra $\mathfrak{g}_{2} \oplus \mathfrak{s u}(2)$.

The same reasoning applies to all $\mathcal{N}=2$ theories without hyper multiplets: these have to be extended with an additional compact $S U(2) / S U(2)$ factor, and therefore reduce to a semi-simple coset in three dimensions. Very-extended simple algebras like $\mathfrak{g}_{2}^{+++}$should not be associated to an $\mathcal{N}=2$ theory. In the conclusions we will discuss whether they correspond to theories with less than eight supercharges.

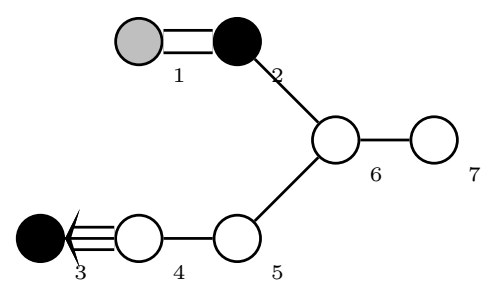

Figure 6: $\left(\mathfrak{g}_{2} \oplus \mathfrak{s u}(2)\right)^{+++}$decomposed as $\mathfrak{s l}(5) \oplus \mathfrak{s u}(2)$, corresponding to a $D=5$ theory with an $S U(2)$ internal symmetry group. Node 1 should be taken compact (see text).

Let us now check whether very-extended $\left(\mathfrak{g}_{2} \oplus \mathfrak{s u}(2)\right)^{+++}$contains the correct generators. The Dynkin diagram of this very-extended semi-simple algebra is given in figure 6 with the $\mathfrak{s l}(5)$ regular subalgebra indicated, while the corresponding decomposition can be found in table 9 in appendix C. The additional node 1 should be understood to give rise to an internal $S U(2)$ symmetry group in $D=5$. Therefore the relevant real form of this algebra is not maximally non-compact. As the largest part of this paper deals with algebras of maximally non-compact, or split, real form, we prefer not introduce the additional notation for other real forms. This can be found in e.g. [17, 58]. Instead we will indicate the consequences of the non-split form in what follows. 
A straightforward comparison between the results for $\left(\mathfrak{g}_{2} \oplus \mathfrak{s u}(2)\right)^{+++}$and $\left(\mathfrak{g}_{2} \oplus \mathfrak{f}_{4}\right)^{+++}$ shows that the results are completely analogous and will therefore not be listed. In particular, one finds exactly the same generators which are mentioned in section 3.2. where now instead of the adjoint of $\mathfrak{f}_{4}$ one finds the adjoint of $\mathfrak{s u}(2)$. Note that the corresponding scalars are all pure gauge since the internal symmetry is compact. In addition there will be three linear constraints on the field strengths of the dual three-forms. In this way one indeed recovers the correct physical field content of the graviton multiplet. Moreover, the three- and four-forms transforming in the adjoint of $\mathfrak{s u}(2)$ coincide exactly with the results of [36]. A puzzle arises for the five-forms, which were not discussed in [36] but are present in the very-extended algebra. This can be seen as a prediction of our proposal for very-extended semi-simple Lie algebras. As we show in appendix A, it is indeed possible to include an $S U(2)$-triplet of five-forms as well, provided they have rather unusual supersymmetry transformations. Hence this example confirms both the need to include an $S U(2) / S U(2)$ factor and our proposal for the extended semi-simple algebras.

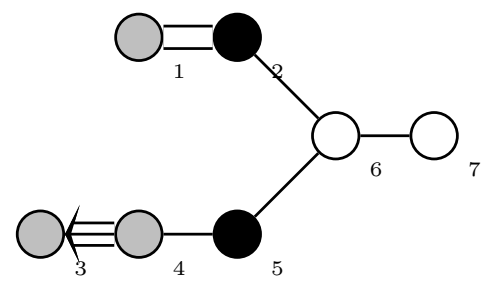

Figure 7: $\left(\mathfrak{g}_{2} \oplus \mathfrak{s u}(2)\right)^{+++}$decomposed as $\mathfrak{s l}(3) \oplus \mathfrak{g}_{2} \oplus \mathfrak{s u}(2)$, corresponding to a $D=3$ theory with an $G_{2} \times S U(2)$ internal symmetry group. Node 1 should be taken compact (see text).

Again we also consider the three-dimensional theory associated to the very-extended algebra. The corresponding Dynkin diagram is given in figure 7 while the decomposition can be found in table 10. It can be verified that this gives rise to the correct physical degrees of freedom: the graviton and scalars in the adjoints of $\mathfrak{g}_{2}$ and $\mathfrak{s u}(2)$, and their duals. In addition the very-extended algebra contains the following two- and three-forms:

- two-forms in the $\left(\mathfrak{g}_{2}, \mathfrak{s u}(2)\right)$ representations $(\mathbf{1} \oplus \mathbf{1} \oplus \mathbf{2 7}, \mathbf{1}) \oplus(\mathbf{1 4}, \mathbf{3})$,

- three-forms in the $\left(\mathfrak{g}_{2}, \mathfrak{s u}(2)\right)$ representations $(\mathbf{1 4} \oplus \mathbf{1 4} \oplus \mathbf{2 7} \oplus \mathbf{6 4}, \mathbf{1}) \oplus(\mathbf{1} \oplus \mathbf{1 4} \oplus$ $14 \oplus \mathbf{2 7} \oplus \mathbf{7 7}, \mathbf{3})$.

The two-forms in this algebra are in perfect agreement with the components of the embedding tensor and thus with the possible gaugings [54]. This is a further confirmation of our proposal.

Note that, in contrast to the $\left(\mathfrak{g}_{2} \oplus \mathfrak{f}_{4}\right)^{+++}$case discussed in the previous section, the present theory does not allow for an alternative uplift. In other words, if we would interchange the vector and hyper multiplets in (8) then the theory does not allow for an uplift to $D \geq 4$. The reason is that the scalars of $\mathcal{M}_{V}$ have to provide the degrees of freedom for e.g. the metric in higher dimensions. The scalar manifold $S U(2) / S U(2)$, 
however, does not have any degrees of freedom associated to it. Therefore with this interpretation the theory only lives in $D=3$. From the point of view of the Dynkin diagram in figure 6 one might think that there is an alternative $\mathfrak{s l}(4)$ regular subalgebra that includes the affine extension node of $\mathfrak{s u}(2)$, but this is not possible due to the nonsplit form; in the theory of group oxidation, the $\mathfrak{s l}(D)$ regular subalgebra is not allowed to be connected to the compact node 1 [58].

\section{Relation to $E_{11}$}

In this section we show that our proposal is consistent with the possibility to obtain certain $\mathcal{N} \leq 2$ theories as truncation of the maximal theory. In particular we will focus on the pure and coupled $\mathcal{N}=2$ theories, both of which have an $\mathcal{N}=8$ origin $[26,37,52]$. We assume that maximal theories are described by an $E_{8}^{+++} \equiv E_{11}$ symmetry (in split form) and will verify that the quotients discussed in the preceding sections arise as subalgebras of $\mathfrak{e}_{11}$. In order to make the analysis rigorous we first review some facts about $\mathfrak{e}_{11}$ and then move on to proving $\left(\mathfrak{g}_{2} \oplus \mathfrak{f}_{4}\right)^{+++} \subseteq \mathfrak{e}_{11}$ and $\left(\mathfrak{g}_{2} \oplus \mathfrak{s u}(2)\right)^{+++} \subseteq \mathfrak{e}_{11}$.

\section{1 $\mathfrak{e}_{11}$ in $\mathfrak{s l}(11)$ basis}

The Dynkin diagram of $\mathfrak{e}_{11}$ is shown in fig. 8 where the exceptional node has been marked as deleted so that there is an $\mathfrak{s l}(11)$ gravity line corresponding to a theory in $D=11$. At level $(l)$ the spectrum contains $[8,11]$ :

- (0): the traceless part of the metric, carrying 120 fields of which 55 will be eliminated due to the local Lorentz symmetry,

- (0): a scalar which provides the trace of the metric and turning $\mathfrak{s l}(11)$ into $\mathfrak{g l}(11)$,

- (1): a three-form as present in $D=11$ supergravity,

- (2): a six-form, which is interpreted as dual to the three-form,

- (3): a generator with mixes properties, interpreted as the dual graviton.

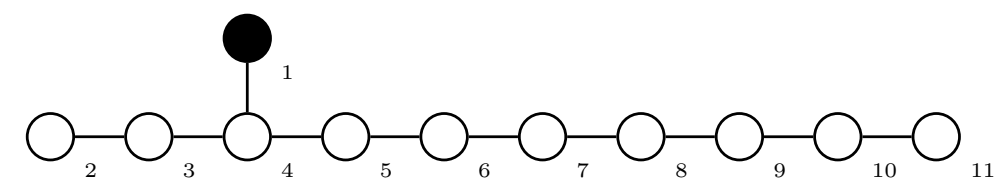

Figure 8: $\mathfrak{e}_{11}$ decomposed as $\mathfrak{s l}(11)$, corresponding to the $D=11$ maximal theory.

We will use the following notation for the generators on levels (0) and (1):

$$
\begin{aligned}
\mathfrak{g l}(11) & : K_{b}^{a}, \quad a, b=1, \ldots, 11, \\
\text { three-form } & : \quad E^{a_{1} a_{2} a_{3}}=E^{\left[a_{1} a_{2} a_{3}\right]} .
\end{aligned}
$$


They commute according to $[8,11]$

$$
\begin{aligned}
{\left[K^{a}{ }_{b}, K_{d}^{c}\right] } & =\delta_{b}^{c} K_{d}^{a}-\delta_{d}^{a} K_{d}^{c}, \\
{\left[K_{b}^{a}, E^{c_{1} c_{2} c_{3}}\right] } & =3 \delta_{b}^{\left[c_{1}\right.} E^{\left.c_{2} c_{3}\right] a} .
\end{aligned}
$$

The Lie algebra $\mathfrak{e}_{11}$ is defined in terms of simple Chevalley generators $e_{i}, f_{i}$ and $h_{i}$ for $i=1, \ldots, 11$ with the relations [39]

$$
\begin{aligned}
{\left[h_{i}, h_{j}\right] } & =0, & {\left[e_{i}, f_{j}\right] } & =\delta_{i j} h_{j}, \\
{\left[h_{i}, e_{j}\right] } & =A_{i j} e_{j}, & {\left[h_{i}, f_{j}\right] } & =-A_{i j} f_{j}, \\
\left(\operatorname{ad} e_{i}\right)^{1-A_{i j}} e_{j} & =0, & \left(\operatorname{ad} f_{i}\right)^{1-A_{i j}} f_{j} & =0 .
\end{aligned}
$$

where $A_{i j}$ are the entries of the Cartan matrix of $\mathfrak{e}_{11}$. The generators $h_{i}$ and $e_{i}$ can be chosen to be related to the basis we chose above by $[9,11]$

$$
\begin{aligned}
& h_{i}=K^{13-i}{ }_{13-i}-K^{12-i}{ }_{12-i} \quad(\text { for } i=2, \ldots, 11), \\
& h_{1}=-\frac{1}{3} \sum_{a=1}^{11} K_{a}^{a}+K^{9}{ }_{9}+K^{10}{ }_{10}+K^{11}{ }_{11}, \\
& e_{i}=K^{13-i}{ }_{12-i} \quad(\text { for } i=2, \ldots, 11), \quad e_{1}=E^{91011}, \\
& f_{i}=K^{12-i}{ }_{13-i} \quad(\text { for } i=2, \ldots, 11), \quad f_{1}=F_{91011}=\left(E^{91011}\right)^{T} .
\end{aligned}
$$

\section{$4.2\left(\mathfrak{g}_{2} \oplus \mathfrak{f}_{4}\right)^{+++} \subset \mathfrak{e}_{11}$}

To show that $\left(\mathfrak{g}_{2} \oplus \mathfrak{f}_{4}\right)^{+++}$as defined in section 2 is a subalgebra of $\mathfrak{e}_{11}$ we exhibit defining Chevalley generators $H_{i}$ and $E_{i}$ (for $i=1, \ldots, 10$ ) as combinations of $\mathfrak{e}_{11}$ generators (12) such that they obey the relations (111) but now with the Cartan matrix $A_{i j}$ encoded in diagram 2. This will describe the derived algebra needed in the construction of $\left(\mathfrak{g}_{2} \oplus\right.$ $\left.\mathfrak{f}_{4}\right)^{+++}$; the quotient by the centre will follow from the fact that the central element corresponds to the zero element of $\mathfrak{e}_{11}$ and therefore is not a linearly independent Cartan generator.

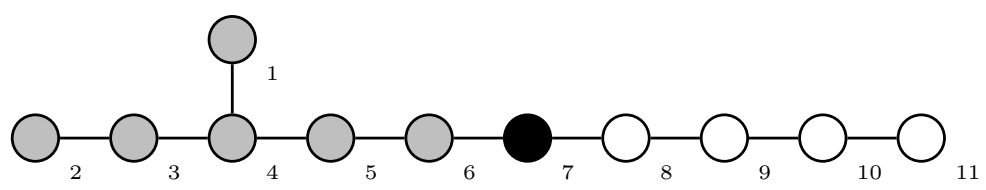

Figure 9: $\mathfrak{e}_{11}$ decomposed as $\mathfrak{s l}(5) \oplus \mathfrak{e}_{6}$, corresponding to the $D=5$ maximal theory with an $E_{6}$ internal symmetry group.

In order to obtain diagram 2 it is most convenient to also take the $D=5$ version of the $\mathfrak{e}_{11}$ diagram. This is shown in figure 9, where the global $E_{6}$ symmetry of the scalar manifold of maximal ungauged supergravity in $D=5$ is evident. The $F_{4}$ symmetry describing the scalar of the truncated coupled theory discussed in section 3.2 is the maximal subgroup $F_{4} \subset E_{6}$ whose defining Lie algebra generators we will give explicitly below. In addition 
we need to find an $F_{4}$ singlet vector generator which gives node 6 of diagram 2 and an $F_{4}$ adjoint three-form which gives node 5 of that diagram.

The correct choices for the Cartan generators are

$$
H_{10}=h_{11}, \quad H_{9}=h_{10}, \quad H_{8}=h_{9}, \quad H_{7}=h_{8},
$$

since the gravity generators are common in both theories,

$$
H_{4}=h_{1}, \quad H_{3}=h_{4}, \quad H_{2}=h_{3}+h_{5}, \quad H_{1}=h_{2}+h_{6},
$$

since this is the correct embedding of $\mathfrak{f}_{4} \subset \mathfrak{e}_{6}$ and

$$
\begin{aligned}
H_{6} & =3 h_{7}+4 h_{6}+5 h_{5}+6 h_{4}+4 h_{3}+2 h_{2}+3 h_{1} \\
& =-K^{1}-K^{2}{ }_{2}-K^{3}{ }_{3}-K^{4}{ }_{4}+2 K^{5}{ }_{5}, \\
H_{5} & =h_{9}+2 h_{8}+3 h_{7}+3 h_{6}+3 h_{5}+3 h_{4}+2 h_{3}+h_{2}+h_{1} \\
& =-\frac{1}{3} \sum_{a=1}^{11} K^{a}{ }_{a}+K^{3}{ }_{3}+K^{4}{ }_{4}+K^{5}{ }_{5}
\end{aligned}
$$

for the connecting nodes. This choice becomes clearer with the expression for the simple positive step operators

$$
E_{10}=e_{11}, \quad E_{9}=e_{10}, \quad E_{8}=e_{9}, \quad E_{7}=e_{8},
$$

again since the gravity sectors are common in both theories,

$$
E_{4}=e_{1}, \quad E_{3}=e_{4}, \quad E_{2}=e_{3}+e_{5}=K_{8}^{7}+K^{9}{ }_{10}, \quad E_{1}=e_{2}+e_{6}=K^{6}{ }_{7}+K^{10}{ }_{11},
$$

from the embedding of $\mathfrak{f}_{4} \subset \mathfrak{e}_{6}$ and

$$
\begin{aligned}
& E_{6}=E^{5611}-E^{5710}+E^{589}, \\
& E_{5}=E^{345} .
\end{aligned}
$$

The generators $F_{i}$ are the transposes of the $E_{i}$ given here.

With the formulæ (13) $-(18)$ one verifies the relations (11), for example

$$
\left[H_{6}, E_{6}\right]=2 E_{6}, \quad\left[H_{7}, E_{6}\right]=-E_{6}, \quad\left[H_{6}, E_{7}\right]=-3 E_{7}, \text { etc. }
$$

For the verification of the Serre relations it is useful to notice that all simple step operators belong $\mathfrak{s l}(2)$ subalgebras and the computations can be shortened by using the representation theory of $\mathfrak{s l}(2)$; otherwise some of the Serre relations involve $\mathfrak{e}_{11}$ commutators up to $\mathfrak{s l}(11)$ level $l=5$ which exceeds the level to which the relations have been worked out 18

An important observation regarding the new Cartan generators $H_{i}$ of (13)-(15) is that they are not all linearly independent: The combinations

$$
\begin{aligned}
c_{\mathfrak{g}_{2}} & =H_{6}+2 H_{7}+H_{8} \\
& =h_{9}+2 h_{8}+3 h_{7}+4 h_{6}+5 h_{5}+6 h_{4}+4 h_{3}+2 h_{2}+3 h_{3}, \\
c_{\mathfrak{f}_{4}} & =H_{1}+2 H_{2}+3 H_{3}+2 H_{4}+H_{5} \\
& =h_{9}+2 h_{8}+3 h_{7}+4 h_{6}+5 h_{5}+6 h_{4}+4 h_{3}+2 h_{2}+3 h_{3}
\end{aligned}
$$

\footnotetext{
${ }^{18}$ The highest known commutators for $\mathfrak{e}_{10}$ involve level $l=4[59]$.
} 
are identical so that $c_{\mathfrak{g}_{2}}-c_{\mathfrak{f}_{4}}=019$ The combinations above were chosen because $c_{\mathfrak{g}_{2}}$ is the central charge of $\mathfrak{g}_{2}^{+}$and $c_{\mathfrak{f}_{4}}$ that of $\mathfrak{f}_{4}^{+}$contained in the diagram. Therefore, $c_{\mathfrak{g}_{2}}-c_{\mathfrak{f}_{4}}$ is precisely the combination that has to be quotiented out of the abstract (derived) algebra generated by $H_{i}, E_{i}$ and $F_{i}$ according to our definition of section 2. This is realised here automatically in $\mathfrak{e}_{11}$ since this combination corresponds to the zero element in $\mathfrak{e}_{11}$. Hence we have shown that the quotient Lie algebra $\left(\mathfrak{g}_{2} \oplus \mathfrak{f}_{4}\right)^{+++}$is a subalgebra of the KacMoody algebra $\mathfrak{e}_{11}$. Therefore any $E_{11}$ invariant dynamics describing $D=11$ supergravity (or an extension thereof) would entail a consistent truncation to the $\mathcal{N}=2$ theory with seven hypermultiplets invariant under the algebra described in section 3.2 .

The definitions (13)-(18) also show the $D=11$ origin of the fields of the $\mathcal{N}=2$ model in $D=5$ : The vector of the gravity multiplet corresponds to the $F_{4}$ invariant combination of the 27 vector fields one obtains from the three-form of $D=11$, whereas the scalars are obtained by the truncation of the $E_{6}$ scalar coset to the $F_{4}$ scalar coset.

\section{$4.3 \quad\left(\mathfrak{g}_{2} \oplus \mathfrak{s u}(2)\right)^{+++} \subset \mathfrak{e}_{11}$}

Here we show that the quotient algebra $\left(\mathfrak{g}_{2} \oplus \mathfrak{s u}(2)\right)^{+++}$, which was discussed in section 3.3 in relation to pure and simple $\mathcal{N}=2$ supergravity in $D=5$, is a subalgebra of $\mathfrak{e}_{11}$. This can be seen most easily by using the result of the preceding section that $\left(\mathfrak{f}_{4} \oplus \mathfrak{g}_{2}\right)^{+++}$is a subalgebra of $\mathfrak{e}_{11}$ and embedding $\left(\mathfrak{g}_{2} \oplus \mathfrak{s u}(2)\right)^{+++}$in $\left(\mathfrak{f}_{4} \oplus \mathfrak{g}_{2}\right)^{+++}$.

We recall that the local part of the internal symmetries of the coupled theory is $S p(3) \times S U(2)$, the compact subgroup of $F_{4}$ (in split form). The $S U(2)$ factor is the Rsymmetry group in $D=5$. It is natural to decompose all $\mathfrak{f}_{4}$ representations, which appear in the level decomposition of $\left(\mathfrak{f}_{4} \oplus \mathfrak{g}_{2}\right)^{+++}$, under $\mathfrak{s p}(3) \oplus \mathfrak{s u}(2)$. Inspection of table 5 shows that the only $\mathfrak{f}_{4}$ representations occurring for fields with at most five space-time indices are the $\mathbf{1}$ and the $\mathbf{5 2}$ of $\mathfrak{f}_{4}$. They decompose as

$$
\begin{aligned}
1 & \rightarrow(1,1) \\
52 & \rightarrow(1,3) \oplus\left(\mathbf{1 4}_{s}, 2\right) \oplus(\mathbf{2 1}, \mathbf{1})
\end{aligned}
$$

under $\mathfrak{s p}(3) \oplus \mathfrak{s u}(2) \subset \mathfrak{f}_{4}$. The restriction to $\mathfrak{s p}(3)$ singlets leaves only $\mathfrak{s u}(2)$ singlets and triplets. Performing this restriction to $\mathfrak{s p}(3)$ invariant generators within $\left(\mathfrak{f}_{4} \oplus \mathfrak{g}_{2}\right)^{+++}$on the first levels yields precisely the fields with at most five space-time indices transforming under $\mathfrak{s u}(2)$ as those of table 9, which lists the lowest levels of $\left(\mathfrak{g}_{2} \oplus \mathfrak{s u}(2)\right)^{++}$decomposed with respect to $\mathfrak{s l}(5) \oplus \mathfrak{s u}(2)$.

The restriction to $\mathfrak{s p}(3)$ invariant states in $\left(\mathfrak{f}_{4} \oplus \mathfrak{g}_{2}\right)^{+++}$defines a subalgebra $\mathfrak{s}$. Since the fundamental generators of $\left(\mathfrak{g}_{2} \oplus \mathfrak{s u}(2)\right)^{+++}$on levels $(0,1)$ and $(1,0)$ are contained in this subalgebra, we deduce that $\left(\mathfrak{g}_{2} \oplus \mathfrak{s u}(2)\right)^{+++} \subset\left(\mathfrak{f}_{4} \oplus \mathfrak{g}_{2}\right)^{+++} \subset \mathfrak{e}_{11} 20$ The embedding into $\mathfrak{e}_{11}$ could also be carried out directly by decomposing all generators of $\mathfrak{e}_{11}$ under $\mathfrak{s l}(5) \oplus \mathfrak{s p}(3) \oplus \mathfrak{s u}(2)$ and then restricting to $\mathfrak{s p}(3)$ singlets. For the fields with up to five space-time indices the invariant generators are identical to those of $\left(\mathfrak{g}_{2} \oplus \mathfrak{s u}(2)\right)^{+++}$

\footnotetext{
${ }^{19}$ We note also that both are identical to the central element of $\mathfrak{e}_{8}^{+} \subset \mathfrak{e}_{11}$.

${ }^{20}$ That the inclusion relation does not only hold at the level of generators but also at the level of Lie brackets follows from the Serre relations which can be deduced for the invariant generators from the embedding.
} 
but more branchings of the type (21) are required to make this manifest, giving a less transparent derivation.

It is of interest to ask whether the subalgebra $\mathfrak{s}$ of $\left(\mathfrak{f}_{4} \oplus \mathfrak{g}_{2}\right)^{+++}$defined by $\mathfrak{s p}(3)$ invariance is identical to $\left(\mathfrak{g}_{2} \oplus \mathfrak{s u}(2)\right)^{+++}$. For the fields with up to five space-time indices there is no difference but one can check by computer analysis that $\left(\mathfrak{g}_{2} \oplus \mathfrak{s u}(2)\right)^{+++}$is in fact a proper subalgebra of $\mathfrak{s}$. By virtue of general characterisation theorems the complexified subalgebra $\mathfrak{s}$ is a Borcherds algebra [60] 21] Since to date generally only generators with at most $D$ indices in a decomposition under a $\mathfrak{s l}(D)$ subalgebra have a supergravity interpretation, we cannot offer an explanation of this difference here (see [62] for a discussion of this point).

The real form of $\left(\mathfrak{g}_{2} \oplus \mathfrak{s u}(2)\right)^{+++}$follows also from the embedding above: Because the scalars at level $(0,0)$ inside the split $\left(\mathfrak{f}_{4} \oplus \mathfrak{g}_{2}\right)^{+++}$which are invariant under $\mathfrak{s p}(3)$ all belong to the compact $\mathfrak{s u}(2) \subset \mathfrak{f}_{4}$, it follows that the $A_{1}$ node of the diagram of $\left(\mathfrak{g}_{2} \oplus \mathfrak{s u}(2)\right)^{+++}$ has to be compact so that the associated summand in the extension process is the compact $\mathfrak{s u}(2)$.

The construction of $\left(\mathfrak{g}_{2} \oplus \mathfrak{s u}(2)\right)^{+++}$inside $\left(\mathfrak{f}_{4} \oplus \mathfrak{g}_{2}\right)^{+++} \subset \mathfrak{e}_{11}$ also gives a $D=11$ origin to the propagating fields of pure $\mathcal{N}=2$ supergravity in $D=5$ as well as of its possible deformations.

We expect that a similar analysis of the quotient algebra can be carried out in all cases when the $\mathcal{N} \leq 2$ theory has a $D=11$ origin.

\section{Discussion}

In this paper we have discussed the extended semi-simple symmetries that play a role in $\mathcal{N} \leq 2$ supergravity. In particular, we have put forward a proposal for the extension of semi-simple Lie algebras, corresponding to the well-known Kac-Moody extensions of simple Lie algebras and based on analogous reasoning leading to the affine extension as the Geroch group. In addition we have argued that an $S U(2) / S U(2)$ scalar manifold has to be coupled to all $\mathcal{N}=2$ theories without hyper multiplets, such that these fall in the semi-simple realm as well. Support for these conjectures has been gathered from a number of different points of view:

- The extended semi-simple Lie algebras give rise to the correct physical degrees of freedom in the $\mathcal{N}=2$ supergravity examples discussed.

- They contain the correct generators corresponding to the non-propagating deformation potentials. In particular, very-extended $\mathfrak{g}_{2} \oplus \mathfrak{f}_{4}$ contains the correct deformation potentials for the coupled theory in $D=5$, corresponding to the gauging of a single isometry of the scalar manifold of the hyper multiplets. Very-extended $\mathfrak{g}_{2} \oplus \mathfrak{s u}(2)$ reproduces the results of [36] on the deformation potentials of the pure theory in $D=5$, corresponding to the gauging of a $U(1) \subset S U(2)$ of the R-symmetry group.

\footnotetext{
${ }^{21}$ This is similar to the way in which the pure $\mathcal{N}=4$ algebra $\mathfrak{d}_{8}^{++}{ }^{+}$is contained in a Borcherds subalgebra of $\mathfrak{e}_{11}$ [61]. There the Borcherds algebra can be constructed by keeping only tensor representations of a $\mathfrak{d}_{10}$ common to $\mathfrak{e}_{11}$ and $\mathfrak{d}_{8}^{+++}$whereas here we have a stronger restriction to $\mathfrak{s p}(3)$ invariant tensors.
} 
Finally, both very-extended algebras contain the correct deformation potentials in $D=3$, where the gaugings have been classified in [54].

- Very-extended $\mathfrak{g}_{2} \oplus \mathfrak{s u}(2)$ predicts the possibility to include a triplet of top-forms in $D=5$, which is checked successfully in appendix A. The supersymmetry variations of these five-forms seem to be of a novel type.

- Both very-extended (quotient) algebras are subalgebras of $\mathfrak{e}_{11}$, corresponding to the truncation of $\mathcal{N}=8$ supergravity to the associated $\mathcal{N}=2$ theories.

We would like to emphasise that our results can be applied to all semi-simple $D=3$ cosets. As we have argued, the homogeneous scalar manifolds will generically be semisimple with two simple factors for $\mathcal{N}=2$ supergravity. In addition, the simple algebras have to be augmented with an $\mathfrak{s u}(2)$ factor.

An additional illustration of the latter is provided by pure $\mathcal{N}=2$ supergravity in four dimensions. Upon reduction to three dimensions this gives rise to the coset

$$
\mathcal{M}_{V}=\frac{S U(2,1)}{S(U(2) \times U(1))} .
$$

The point is that $\mathfrak{s u}(2,1)^{+++}$does not contain any three-forms when decomposed with respect to $S L(4)$ and hence predicts no gaugings of this four-dimensional supergravity. However, it is known that one can gauge a $U(1)$ in the $S U(2)$ part of the R-symmetry group [63,64]. One can not gauge the separate $U(1)$ factor of $H_{\mathrm{R}}=S U(2) \times U(1)$ as the vector and its Hodge dual transform as a doublet under it. We have checked that very-extended $\mathfrak{s u}(2,1) \oplus \mathfrak{s u}(2)$ contains deformation potentials transforming as an $S U(2)$ triplet and a $U(1)$ doublet, consistent with this gauging. In addition it predicts an $S U(2)$ triplet of two-forms with vanishing field strengths and two $S U(2)$ triplets of four-forms in this theory. It would be interesting to include these explicitly, similar to [36].

We expect the same phenomenon of extended semi-simple Lie algebras to play a role in $\mathcal{N}=1$ supergravities. These theories reduce to a Kähler scalar manifold 22 in three dimensions [66,67], homogeneous examples of which can be found in e.g. [68]. An important point is that the product of two Kähler manifolds is again a Kähler manifold. Therefore one can couple any number of homogeneous spaces to $\mathcal{N}=1$ supergravity. Such products appear naturally in e.g. the truncation from $\mathcal{N}=2$ to $\mathcal{N}=1$ [69]. Hence, for $\mathcal{N}=1$ supergravity, in three dimensions one can have semi-simple algebras of isometries with more than two factors: $\mathfrak{g}_{a} \oplus \mathfrak{g}_{b} \oplus \mathfrak{g}_{c} \oplus \ldots$ The extensions of such semi-simple algebras were outlined in section 2 ,

Due to the sum of simple factors in $\mathfrak{g}$, one can argue that the algebra of isometries of homogeneous scalar manifolds is generically semi-simple for $\mathcal{N}=1$ supergravity. This is similar to what we found for $\mathcal{N}=2$ supergravity. However, the $\mathcal{N}=2$ situation is different in that one can not add any number of homogeneous spaces, as the product of quaternionic-Kähler spaces is not quaternionic-Kähler itself (except when they are hyperKähler spaces, which are not allowed in $\mathcal{N}=2$ supergravity). Hence one can only couple two such spaces, associated with $\mathcal{N}=2$ vector and hyper multiplets.

\footnotetext{
${ }^{22}$ The additional topological constraint requiring it to be Hodge-Kähler [65] is not important here.
} 
An example of a semi-simple algebra appears in $\mathcal{N}=1, D=4$ supergravity coupled to a chiral multiplet. After reduction to three dimensions this theory corresponds to the coset

$$
\frac{G}{H}=\frac{S L(2)}{S O(2)} \times \frac{S L(2)}{S O(2)}
$$

In line with the previous discussion it consists of a product of Kähler manifolds. The hidden symmetry group is therefore semi-simple: $S L(2) \times S L(2) \simeq S O(2,2)$. This theory and the very-extended algebra $(\mathfrak{s l}(2) \oplus \mathfrak{s l}(2))^{+++}$are discussed in detail in appendix $\mathrm{B}$. Based on covariance with respect to $\mathfrak{s o}\left(2,2+n_{\mathrm{V}}\right)$, we confirm that the very-extended semi-simple algebra $(\mathfrak{s l}(2) \oplus \mathfrak{s l}(2))^{+++}$contains exactly the generators one would expect.

However, in addition to the appearance of semi-simple symmetries, we also expect a number of new features to appear for $\mathcal{N}=1$. This can be seen from the three-dimensional classification of [54]. A first point is that one has to incorporate the $S O(2)$ R-symmetry group as a central extension of the isometry group. Furthermore, in contrast to $\mathcal{N} \geq 2$, there is no linear constraint on the possible gaugings for $\mathcal{N}=1$. Finally, there are deformations that do not correspond to a gauging but rather to the addition of a superpotential. Therefore we would not expect e.g. $(\mathfrak{s l}(2) \oplus \mathfrak{s l}(2))^{+++}$to describe all the deformations of the $\mathcal{N}=1$ theory. It remains to be seen to what extent all $\mathcal{N}=1$ features can be reproduced from the Kac-Moody side.

Similarly, one could consider supergravity theories with six supersymmetries. These theories have a single quaternionic-Kähler manifold in three dimensions [25]. The Rsymmetry group is $H_{\mathrm{R}}=S O(3)$ and hence these do not suffer from the R-symmetry problems discussed. Therefore one could expect that very-extended simple Lie algebras, such as $\mathfrak{g}_{2}^{+++}$, incorporate both the physical and the non-propagating degrees of freedom of this theory. However, it turns out that for these theories there is no linear constraint on the possible gaugings either [54] and hence the Kac-Moody correspondence remains unclear as well.

Finally, the discussion so far has been concerned with the correspondence between supergravity and (quotients of) Kac-Moody algebras to $\mathcal{N} \leq 2$ theories with homogeneous spaces. More specifically, we have restricted ourselves to homogeneous scalar manifolds with semi-simple groups of isometries. Interesting future research would be to investigate the extended algebras associated to homogeneous but non-semi-simple groups, or even to venture into the realm of non-homogeneous scalar manifolds.

Besides these interesting points regarding the correspondence for the bosonic sectors of various supergravity theories it would be worthwhile to extend our new cases also to the fermionic sector. It is known that the maximally supersymmetric theories in $D=11$ and $D=10$ have propagating fermionic degress of freedom which can be grouped into finitedimensional (unfaithful) representations of $K\left(E_{11}\right)[33,34,70]$ and also the half-maximal case has been analysed [71]. We strongly expect that the compact subalgebras of the quotient algebras presented here will also admit finite-dimensional spinor representations which correspond to the fermionic degrees of freedom of the various $\mathcal{N} \leq 2$ theories they belong to. 


\section{Acknowledgements}

We thank Eric Bergshoeff, Alessio Celi, Alex Feingold, Joaquim Gomis, Marc Henneaux, Hermann Nicolai, Teake Nutma, Henning Samtleben, Igor Schnakenburg and Antoine van Proeyen for very stimulating discussions. In addition, we acknowledge using the SimpLie program of Teake Nutma for the decompositions of appendix C. The authors would like to thank each other's institutions for hospitality. A.K. is a Research Associate of the Fonds de la Recherche Scientifique-FNRS, Belgium. The work of D.R. has been supported by the European EC-RTN project MRTN-CT-2004-005104, MCYT FPA 2004-04582-C02-01 and CIRIT GC 2005SGR-00564.

\section{A Five-forms in pure and simple $D=5$ supergravity}

In [36] it has been analysed on which higher-rank potentials the supersymmetry algebra of pure and simple $D=5$ supergravity can be analysed. We will briefly review their results for the ungauged case and then show that it is possible to introduce a further extension. All conventions are identical to [36] where more details can be found.

In the standard formulation the supersymmetry algebra is realised on the metric, a gravitino and a vector. Their supersymmetry transformations are given by

$$
\begin{aligned}
\delta e_{\mu}{ }^{m} & =\frac{1}{2} \bar{\epsilon}^{i} \Gamma^{m} \psi_{\mu i}, \\
\delta \psi_{\mu i} & =D_{\mu} \epsilon_{i}+\frac{1}{4 \sqrt{6}} i\left(\Gamma_{\mu}{ }^{\nu \rho}-4 \delta_{\mu}{ }^{\nu} \Gamma^{\rho}\right) F_{\nu \rho} \epsilon_{i}, \\
\delta A_{\mu} & =-\frac{\sqrt{6}}{4} i \bar{\epsilon}^{i} \psi_{\mu i},
\end{aligned}
$$

where $i$ is the $S U(2)$ R-symmetry index. These satisfy the commutator

$$
\left[\delta_{1}, \delta_{2}\right]=\delta_{\text {gct }}+\delta_{\text {Lorentz }}+\delta_{\text {susy }}+\delta_{\text {gauge }}+\delta_{\mathcal{L}}
$$

where the first four terms on the right hand side are transformations with the usual action on the fields and parameters given by

$$
\begin{aligned}
\xi^{\mu}=\frac{1}{2} \bar{\epsilon}_{1}^{i} \Gamma^{\mu} \epsilon_{2 i}, & \Lambda^{m n}=\xi^{\nu} \omega_{\nu}^{m n}+\frac{1}{4 \sqrt{6}} i \bar{\epsilon}_{1}^{i}\left(\Gamma^{m n p q}+4 g^{m p} g^{n q}\right) F_{p q} \epsilon_{2 i}, \\
\eta^{i}=-\xi^{\mu} \psi_{\mu i}, & \lambda^{(0)}=-\frac{\sqrt{6}}{4} i \bar{\epsilon}_{1}^{i} \epsilon_{2 i}-\xi^{\nu} A_{\nu}
\end{aligned}
$$

while $\delta_{\mathcal{L}}$ imposes a possible first-order field equation (e.g. for the gravitino) which takes the form of a duality relation for the bosons.

In [36] it was shown that the supersymmetry algebra can also be realised on higherrank gauge potentials with the following supersymmetry transformations:

$$
\begin{aligned}
\delta B_{\mu \nu} & =b_{1} \bar{\epsilon}^{i} \Gamma_{[\mu} \psi_{\nu] i}+b_{2} A_{[\mu} \delta A_{\nu]} \\
\delta C_{\mu \nu \rho}^{i j} & =i c_{1} \bar{\epsilon}^{(i} \Gamma_{[\mu \nu} \psi_{\rho]}^{j)} \\
\delta D_{\mu \nu \rho \sigma}^{i j} & =d_{1} \bar{\epsilon}^{(i} \Gamma_{[\mu \nu \rho} \psi_{\sigma]}^{j)}+d_{2} A_{[\mu} \delta C_{\nu \rho \sigma]}^{i j},
\end{aligned}
$$


provided $b_{1}=\frac{3}{4} b_{2}=-\frac{1}{2} \sqrt{6}$ and $c_{1} d_{2}=-\sqrt{6} d_{1}$. The latter two are symmetric in their $S U(2)$ indices and subject to the symplectic constraint

$$
C^{i j}-C_{i j}^{*}=D^{i j}-D_{i j}^{*}=0 .
$$

They transform as $S U(2)$ triplets. On the right hand side of the algebra we find the gauge transformations

$$
\begin{array}{cl}
\delta_{\text {gauge }} B_{\mu \nu} & =-2 \partial_{[\mu} \lambda_{\nu]}^{(1)}-\frac{1}{3} \sqrt{6} \lambda^{(0)} F_{\mu \nu}, \quad \lambda_{\nu}^{(1)}=-B_{\nu \sigma} \xi^{\sigma}+\frac{1}{4} \sqrt{6} \bar{\epsilon}_{1}^{i} \Gamma_{\nu} \epsilon_{2 i}-\frac{1}{2} i \bar{\epsilon}_{1}^{i} \epsilon_{2 i} A_{\nu}, \\
\delta_{\text {gauge }} C_{\mu \nu \rho}^{i j} & =-3 \partial_{[\mu} \lambda_{\nu \rho]}^{(2) i j}, \quad \lambda_{\mu \nu}^{(2) i j}=-C_{\mu \nu \rho}^{i j} \xi^{\rho}+\frac{1}{3} i c_{1} \bar{\epsilon}_{1}^{(i} \Gamma_{\mu \nu} \epsilon_{2}^{j)}, \\
\delta_{\text {gauge }} D_{\mu \nu \rho \sigma}^{i j} & =-4 \partial_{[\mu} \lambda_{\nu \rho \sigma]}^{(3) i j}, \quad \lambda_{\mu \nu \rho}^{(3) i j}=-D_{\mu \nu \rho \sigma}^{i j} \xi^{\sigma}-\frac{1}{4} d_{1}\left(\bar{\epsilon}_{1}^{(i} \Gamma_{\mu \nu \rho} \epsilon_{2}^{j)}-\sqrt{6} i A_{[\mu} \bar{\epsilon}_{1}^{(i} \Gamma_{\nu \rho]} \epsilon_{2}^{j)}\right) .
\end{array}
$$

and the following first-order field equations:

$$
\begin{aligned}
\delta_{\mathcal{L}} B_{\mu \nu} & =-\left(3 \partial_{[\mu} B_{\nu \rho]}-\sqrt{6} A_{[\mu} F_{\nu \rho]}-\frac{1}{2} \sqrt{-g} \varepsilon_{\mu \nu \rho \sigma \lambda} F^{\sigma \lambda}\right) \xi^{\rho}, \\
\delta_{\mathcal{L}} C_{\mu \nu \rho}^{i j} & =-\left(4 \partial_{[\mu} C_{\nu \rho \sigma]}^{i j}\right) \xi^{\sigma}, \quad \delta_{\mathcal{L}} D_{\mu \nu \rho \sigma}^{i j}=-\left(5 \partial_{[\mu} D_{\nu \rho \sigma \tau]}^{i j}\right) \xi^{\tau},
\end{aligned}
$$

The first line identifies $B$ as the dual of $A$ while the second line implies the vanishing of the field strengths of $C^{i j}$ and $D^{i j}$.

As shown in [36], it is impossible to realise supersymmetry on an independent fiveform with a leading term that is bilinear in the gravitino and the supersymmetry parameter. The only such possibility leads to the Levi-Civita tensor and hence a dependent field. However, it is in fact possible to introduce an $S U(2)$ triplet of five-forms whose supersymmetry variation contains only subleading terms that are trilinear in the gravitino, the supersymmetry parameter and a lower-rank gauge potential. Therefore this supersymmetry variation will vanish in a linearised approximation. More precisely, their supersymmetry transformation is given by

$$
\delta E_{\mu \nu \rho \sigma \tau}^{i j}=e_{3} B_{[\mu \nu} \delta C_{\rho \sigma \tau]}^{i j}+e_{4} A_{[\mu} \delta D_{\nu \rho \sigma \tau]}^{i j} .
$$

The supersymmetry algebra closes on these five-forms provided $c_{1} e_{3}=\frac{3}{2} d_{1} e_{4}$ and up to the gauge transformation

$\delta_{\text {gauge }} E_{\mu \nu \rho \sigma \tau}^{i j}=-5 \partial_{[\mu} \lambda_{\nu \rho \sigma \tau]}^{(4) i j}, \quad \lambda_{\mu \nu \rho \sigma}^{(4) i j}=-E_{\mu \nu \rho \sigma \tau}^{(i j)} \xi^{\tau}+\frac{1}{5} d_{1} e_{4}\left(\frac{3}{2} i B_{[\mu \nu} \bar{\epsilon}_{1}^{(i)} \Gamma_{\rho \sigma]} \epsilon_{2}^{j)}+A_{[\mu} \bar{\epsilon}_{1}^{(i)} \Gamma_{\nu \rho \sigma]} \epsilon_{2}^{j)}\right)$.

Note the absence of an independent four-form gauge transformation that is quadratic in the supersymmetry parameters and does not contain other gauge potentials. This follows from the unusual form of the supersymmetry transformations (31). It seems that such transformation properties have not been encountered before and are only possible for space-time filling top-forms.

\section{B A semi-simple example in $\mathcal{N}=1$ supergravity}

\section{B.1 General aspects}

As an illustration of the general discussion in the conclusions we will consider a particular semi-simple algebra that appears naturally in $\mathcal{N}=1$ supergravity. As is well known, 
the bosonic sector of the pure four-dimensional theory reduces to the (Ehlers) scalar coset $S L(2) / S O(2)$ in three dimensions. Many aspects of hidden symmetries were first discussed in this context. Instead of the pure theory in four dimensions we consider the coupling to a chiral multiplet, whose bosonic sector consists of two scalars that can be taken to parametrise the scalar coset $S L(2) / S O(2)$ as well. Consequently we will end up with the product of these two simple factors in three dimensions.

\begin{tabular}{||c||c||c|c||}
\hline$D$ & $H_{\mathrm{R}}$ & $G$ & $H$ \\
\hline \hline 4 & $S O(2)$ & $S O\left(n_{\mathrm{V}}\right) \times S L(2)$ & $S O\left(n_{\mathrm{V}}\right) \times S O(2)$ \\
\hline 3 & $S O(2)$ & $S O\left(2,2+n_{\mathrm{V}}\right)$ & $S O(2) \times S O\left(2+n_{\mathrm{V}}\right)$ \\
\hline
\end{tabular}

Table 3: The global symmetries $G$ and their compact subgroups $H$ of a class of $\mathcal{N}=1$ theories, consisting in four dimensions of the graviton, one chiral and $n_{\mathrm{V}}$ vector multiplets.

It will be useful to extend this theory further by adding $n_{\mathrm{V}}$ vector multiplets with minimal couplings. The symmetries of these theories and their dimensional reductions can be found in table 3. The theory without vectors, i.e. $n_{\mathrm{V}}=0$, can be seen as a natural limit of the generic case with $n_{\mathrm{V}} \geq 1$. However, note that that while $\mathfrak{s o}\left(2,2+n_{\mathrm{V}}\right)$ is semi-simple for $n_{\mathrm{V}}=0$, it is simple for $n_{\mathrm{V}} \geq 1$. Hence the very-extensions of the latter are well known. The corresponding $(D-1)$ - and $D$-form potential 23 are given in table 4 in terms of the global symmetry groups of these theories.

\begin{tabular}{||c||c|c||}
\hline$D$ & $(D-1)$-forms & $D$-forms \\
\hline \hline 4 & $(2, \square) \oplus(2, \boxminus)$ & $(3,1) \oplus(3, \boxminus) \oplus(3, \boxminus) \oplus \boxminus \oplus \boxminus \oplus \boxminus$ \\
\hline 3 & $1 \oplus \square \oplus \boxminus, ~$ & $\square \oplus \boxminus \oplus \boxminus \oplus \boxminus \oplus \boxminus$ \\
\hline
\end{tabular}

Table 4: The deformation and top-form potentials in terms of irreps of $G=S O\left(2,2+n_{\mathrm{V}}\right)$ for this class of $\mathcal{N}=1$ supergravities.

The derivation of the representations in table 4 is not valid for $n_{\mathrm{V}}=0$, since the corresponding Lie algebra is semi-simple: $\mathfrak{s o}(2,2) \simeq \mathfrak{s l}(2) \oplus \mathfrak{s l}(2)$. From the supergravity viewpoint, however, one would of course expect a 'covariant' answer in $n_{V}$. Therefore one has the following consistency check on any proposal for the very-extended $\mathfrak{s l}(2) \oplus \mathfrak{s l}(2)$ : its four- and three-dimensional decompositions should give rise to the $(D-1)$ - and $D$-forms of the above table in the limit where $n_{\mathrm{V}}$ vanishes. We will now discuss this extended semi-simple Lie algebra.

\section{B.2 Very-extended $\mathfrak{s l}(2) \oplus \mathfrak{s l}(2)$ and the $\mathcal{N}=1$ theory}

The decomposition of very-extended $\mathfrak{s l}(2) \oplus \mathfrak{s l}(2)$ with respect to $\mathfrak{s l}(4)$, i.e. in four dimensions, is illustrated in figure10. The resulting list of generators with up to four space-time

\footnotetext{
${ }^{23}$ Note that these are identical to the $(D-1)$ - and $D$-form representations of very-extended $S O(8,8+$ $n_{\mathrm{V}}$ ), corresponding of half-maximal supergravity [20], as these are different real forms of the same algebra.
} 
indices is given in table 10 in appendix $\mathrm{C}$. These can be interpreted to correspond to the following physical degrees of freedom (at level $\left.\left(l_{1}, l_{2}\right)\right)$ :

- $(0,0)$ : the traceless part of the metric, carrying 15 degrees of freedom of which 6 will be eliminated due to the local Lorentz symmetry,

- $(0,0)$ : the $\mathfrak{s l}(2)$ scalars, transforming in the adjoint of the internal symmetry and subject to the local $S O(2)$ symmetry,

- $(0,0)$ : a scalar which provides the trace of the metric,

- $(1,0)$ : a symmetric tensor, which is interpreted as the dual graviton,

- $(0,1)$ : an $\mathfrak{s l}(2)$ triplet of two-forms, which are interpreted as dual to the scalars.

Again one finds scalars and the dual two-forms in the adjoint of the internal symmetry group $S L(2)$, while there are only corresponding two physical degrees of freedom. This is taken care of by the compact $S O(2)$ subgroup, which eliminates one scalar and imposes a linear constraint on the three-form field strengths.

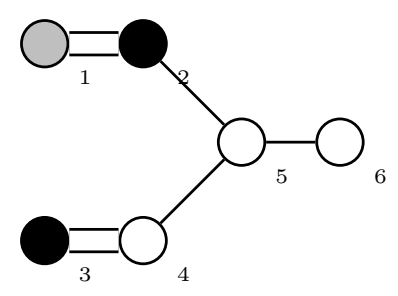

Figure 10: $\quad(\mathfrak{s l}(2) \oplus \mathfrak{s l}(2))^{+++}$decomposed as $\mathfrak{s l}(4) \oplus \mathfrak{s l}(2)$, corresponding to a $D=4$ theory with an $S L(2)$ internal symmetry group.

In this way the above generators exactly coincide with the physical field content. Of the additional generators corresponding to non-propagating degrees of freedom, the purely anti-symmetric ones are

- $(0,2)$ : an $\mathfrak{s l}(2)$ triplet of four-forms,

while the very-extended algebra does not contain any three-forms. These representations of three- and four-forms coincide with the predictions of table 4 for $n_{\mathrm{V}}=0$. As far as we are aware, the possibility to include these four-forms in $\mathcal{N}=1, D=4$ supergravity has not been discussed in the literature.

Next we will discuss the decomposition of $(\mathfrak{s l}(2) \oplus \mathfrak{s l}(2))^{+++}$with respect to $\mathfrak{s l}(3)$, i.e. in three dimensions. The corresponding Dynkin diagram is given in figure 11, while the resulting list of generators can be found in table 11 in appendix C. It can be verified that these lead to exactly the correct physical degrees of freedom of the theory. Again the matching requires the quotienting out of one of the central charges appearing at level $(0,0)$ with multiplicity 2 . In addition, the following anti-symmetric non-propagating degrees of freedom are present in the very-extended algebra: 


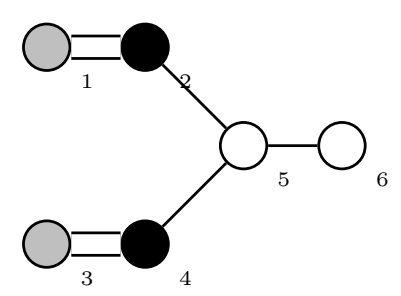

Figure 11: $(\mathfrak{s l}(2) \oplus \mathfrak{s l}(2))^{+++}$decomposed as $\mathfrak{s l}(3) \oplus \mathfrak{s l}(2) \oplus \mathfrak{s l}(2)$, corresponding to a $D=3$ theory with an $S O(2,2)$ internal symmetry group.

- $l=(1,1),(2,0)$ and $(0,2)$ : two-forms in the $\mathfrak{s o}(2,2)$ representations $\mathbf{1} \oplus \mathbf{1} \oplus \mathbf{9}$, - $l=(2,1)$ and $(1,2)$ : three-forms in the $\mathfrak{s o}(2,2)$ representations $\mathbf{3} \oplus \mathbf{3} \oplus \mathbf{9} \oplus \mathbf{9}$.

Comparing with table 4, the representations of two-forms are again correct. The same holds for the three-forms when a subtlety unrelated to the focus of this paper has been taken into account 24 .

\section{Decomposition tables}

Below we give the relevant decompositions with respect to regular $\mathfrak{s l}(D)_{r}$ subalgebras of the Kac-Moody algebras associated to very-extended semi-simple Lie algebras. The number of Cartan subalgebra scalars has been adjusted according to the quotient procedure described in section 2, In all cases we give all the generators at positive levels with up to and including $D$ space-time indices. The tables were obtained by using the SimpLie program [72] and Mathematica code by the first author which was also used for [16].

The different columns in the tables are as follows:

- $l$ is the level in the decomposition,

- $p_{r}$ are the Dynkin labels of the regular (gravity) subalgebra,

- $p_{i}$ are the Dynkin labels of the internal symmetry subalgebra (if present),

\footnotetext{
${ }^{24}$ The general formulae would predict an additional 1. However, an additional requirement on the threeforms is that their $\mathfrak{s o}\left(2,2+n_{\mathrm{V}}\right)$ representations should be contained in the product of the representations of the vectors and two-forms:

$$
\boxminus \otimes(1 \oplus \square \square \oplus)
$$

For $n_{\mathrm{V}}$ large enough this contains the representations of three-forms in table 4 . For $n_{\mathrm{V}}=0$ the antisymmetric four-form representation cannot be generated, however. Hence the missing singlet in veryextended $\mathfrak{s l}(2) \oplus \mathfrak{s l}(2)$ is not a problem: it should not be there, and indeed is not. In fact, a missing top-form could be a more general phenomenon: we are aware that there is a similar mismatch with respect to the generic formulae for $\mathfrak{s o}(4,4)^{+++}$in $D=6$ and $\mathfrak{s o}(4,3)^{+++}$in $D=5$. We thank Eric Bergshoeff and Teake Nutma for discussions on this point.
} 
- 'vector' is the root vector $\alpha$ in the algebra in whose root space the lowest weight vector of the representation lies,

- $\alpha^{2}$ is the norm of the root vector,

- $d_{r}$ is the dimension of the regular subalgebra representation,

- $d_{i}$ is the dimension of the internal subalgebra representation,

- $\mu$ is the outer multiplicity of the representation listed in a given row,

- ind is the number of space-time indices of this representation.

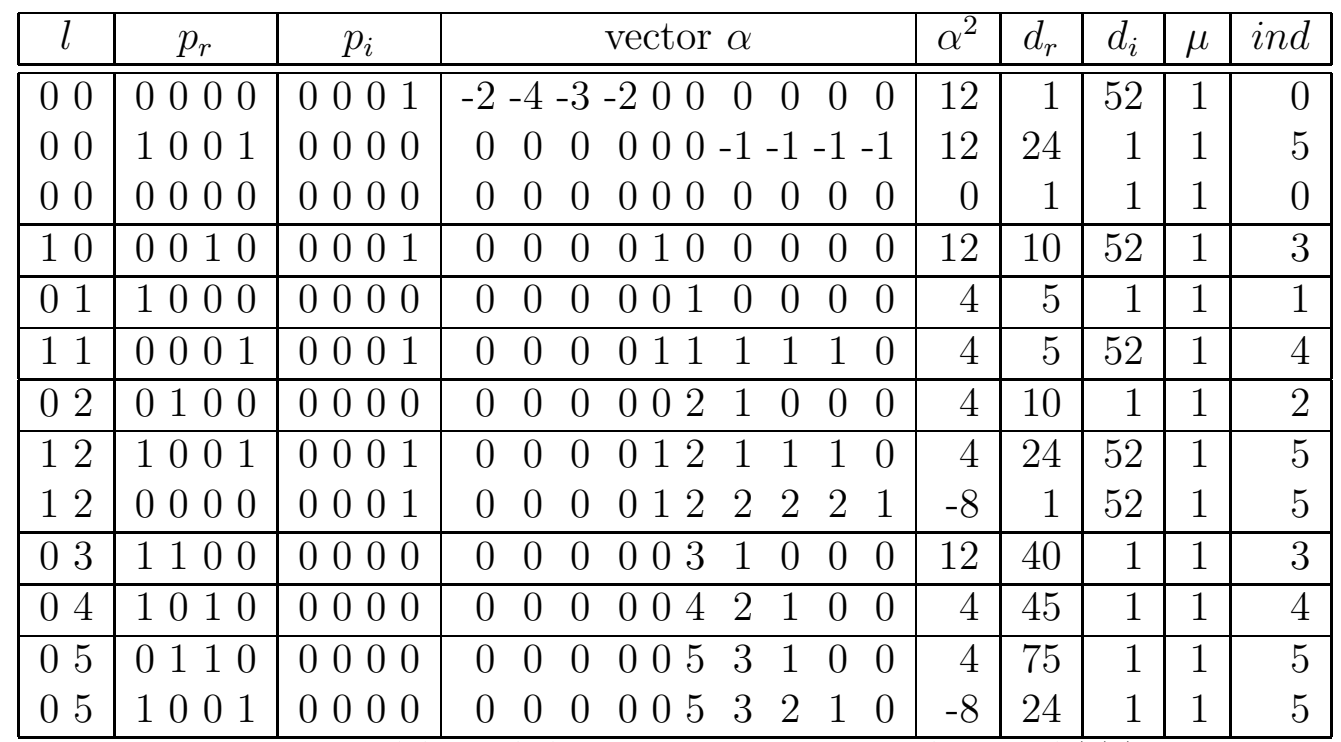

Table 5: $\mathfrak{s l}(5)_{r} \oplus\left(\mathfrak{f}_{4}\right)_{i}$ representations in $\left(\mathfrak{g}_{2} \oplus \mathfrak{f}_{4}\right)^{+++}$

\begin{tabular}{|c|c|c|c|c|c|c|c|c|}
\hline$l$ & $p_{r}$ & $p_{i}$ & vector $\alpha$ & $\alpha^{2}$ & $d_{r}$ & $d_{i}$ & $\mu$ & ind \\
\hline 00 & 00 & 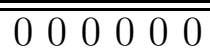 & $\begin{array}{ccccccccccc}0 & 0 & 0 & 0 & 0 & 0 & 0 & 0 & 0 & 0\end{array}$ & 0 & 1 & $\overline{1}$ & 1 & 0 \\
\hline 00 & 11 & 0000000 & $\begin{array}{llllllllll}0 & 0 & 0 & 0 & 0 & 0 & 0 & 0 & -1 & -1\end{array}$ & 12 & 8 & 1 & 1 & 3 \\
\hline 00 & 00 & $\begin{array}{llllll}0 & 0 & 0 & 0 & 0 & 1\end{array}$ & $\begin{array}{lllllllllll}0 & 0 & 0 & 0 & 0 & -3 & -2 & 0 & 0 & 0\end{array}$ & 12 & 1 & 14 & 1 & 0 \\
\hline 00 & 00 & 00010100 & 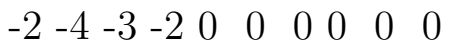 & 12 & 1 & 52 & 1 & 0 \\
\hline 01 & 10 & $\begin{array}{lllllll}0 & 0 & 0 & 0 & 0 & 1\end{array}$ & $\begin{array}{lllllllllll}0 & 0 & 0 & 0 & 0 & 0 & 0 & 1 & 0 & 0 \\
\end{array}$ & 12 & 3 & 14 & 1 & 1 \\
\hline 02 & 01 & $\begin{array}{lllllll}0 & 0 & 0 & 0 & 0 & 0\end{array}$ & $\begin{array}{llllllllll}0 & 0 & 0 & 0 & 0 & 6 & 4 & 2 & 1 & 0\end{array}$ & -12 & 3 & 1 & 1 & 2 \\
\hline 02 & 01 & 0000020 & $\begin{array}{llllllllll}0 & 0 & 0 & 0 & 0 & 2 & 2 & 2 & 1 & 0\end{array}$ & 4 & 3 & 27 & 1 & 2 \\
\hline 02 & 20 & $\begin{array}{llllll}0 & 0 & 0 & 0 & 0 & 1 \\
\end{array}$ & $\begin{array}{llllllllll}0 & 0 & 0 & 0 & 0 & 3 & 2 & 2 & 0 & 0 \\
\end{array}$ & 12 & 6 & 14 & 1 & 2 \\
\hline 10 & 10 & 00001000 & $\begin{array}{llllllllll}0 & 0 & 0 & 0 & 1 & 0 & 0 & 0 & 0 & 0\end{array}$ & 12 & 3 & 52 & 1 & 1 \\
\hline 11 & 01 & $\begin{array}{lllllll} & 0 & 0 & 1 & 0 & 1\end{array}$ & $\begin{array}{lllllllllll}0 & 0 & 0 & 0 & 1 & 0 & 0 & 1 & 1 & 0\end{array}$ & 12 & 3 & 728 & 1 & 2 \\
\hline 20 & 01 & 00000000 & $\begin{array}{llllllllll}4 & 8 & 6 & 4 & 2 & 0 & 0 & 0 & 1 & 0\end{array}$ & -12 & 3 & 1 & 1 & 2 \\
\hline 20 & 20 & 000100 & $\begin{array}{llllllllll}2 & 4 & 3 & 2 & 2 & 0 & 0 & 0 & 0 & 0\end{array}$ & 12 & 6 & 52 & 1 & 2 \\
\hline 20 & 01 & 2000000 & $\begin{array}{llllllllll}0 & 2 & 2 & 2 & 2 & 0 & 0 & 0 & 1 & 0\end{array}$ & 12 & 3 & 324 & 1 & 2 \\
\hline 12 & 00 & 00001000 & $\begin{array}{llllllllll}0 & 0 & 0 & 0 & 1 & 6 & 4 & 2 & 2 & 1\end{array}$ & -24 & 1 & 52 & 1 & 3 \\
\hline
\end{tabular}




\begin{tabular}{|r|r|rr|rrrrrr|rrrrrrrrrr|r|r|r|r|r|}
1 & 2 & 1 & 1 & 0 & 0 & 0 & 1 & 0 & 0 & 0 & 0 & 0 & 0 & 1 & 6 & 4 & 2 & 1 & 0 & -12 & 8 & 52 & 1 & 3 \\
1 & 2 & 0 & 0 & 0 & 0 & 0 & 1 & 0 & 1 & 0 & 0 & 0 & 0 & 1 & 3 & 2 & 2 & 2 & 1 & -12 & 1 & 728 & 1 & 3 \\
1 & 2 & 0 & 0 & 0 & 0 & 0 & 1 & 2 & 0 & 0 & 0 & 0 & 0 & 1 & 2 & 2 & 2 & 2 & 1 & -8 & 1 & 1404 & 1 & 3 \\
1 & 2 & 1 & 1 & 0 & 0 & 0 & 1 & 0 & 1 & 0 & 0 & 0 & 0 & 1 & 3 & 2 & 2 & 1 & 0 & 0 & 8 & 728 & 1 & 3 \\
1 & 2 & 1 & 1 & 0 & 0 & 0 & 1 & 2 & 0 & 0 & 0 & 0 & 0 & 1 & 2 & 2 & 2 & 1 & 0 & 4 & 8 & 1404 & 1 & 3 \\
1 & 2 & 0 & 0 & 0 & 0 & 0 & 1 & 3 & 0 & 0 & 0 & 0 & 0 & 1 & 0 & 1 & 2 & 2 & 1 & 12 & 1 & 4004 & 1 & 3 \\
\hline 2 & 1 & 0 & 0 & 0 & 0 & 0 & 0 & 0 & 1 & 4 & 8 & 6 & 4 & 2 & 0 & 0 & 1 & 2 & 1 & -24 & 1 & 14 & 1 & 3 \\
2 & 1 & 1 & 1 & 0 & 0 & 0 & 0 & 0 & 1 & 4 & 8 & 6 & 4 & 2 & 0 & 0 & 1 & 1 & 0 & -12 & 8 & 14 & 1 & 3 \\
2 & 1 & 0 & 0 & 0 & 0 & 0 & 1 & 0 & 1 & 2 & 4 & 3 & 2 & 2 & 0 & 0 & 1 & 2 & 1 & -12 & 1 & 728 & 1 & 3 \\
2 & 1 & 1 & 1 & 0 & 0 & 0 & 1 & 0 & 1 & 2 & 4 & 3 & 2 & 2 & 0 & 0 & 1 & 1 & 0 & 0 & 8 & 728 & 1 & 3 \\
2 & 1 & 0 & 0 & 2 & 0 & 0 & 0 & 0 & 1 & 0 & 2 & 2 & 2 & 2 & 0 & 0 & 1 & 2 & 1 & 0 & 1 & 4536 & 1 & 3 \\
2 & 1 & 1 & 1 & 2 & 0 & 0 & 0 & 0 & 1 & 0 & 2 & 2 & 2 & 2 & 0 & 0 & 1 & 1 & 0 & 12 & 8 & 4536 & 1 & 3 \\
2 & 1 & 0 & 0 & 0 & 0 & 1 & 0 & 0 & 1 & 0 & 0 & 0 & 1 & 2 & 0 & 0 & 1 & 2 & 1 & 12 & 1 & 17836 & 1 & 3 \\
\hline
\end{tabular}

Table 6: $\mathfrak{s l}(3)_{r} \oplus\left(\mathfrak{f}_{4} \oplus \mathfrak{g}_{2}\right)_{i}$ representations in $\left(\mathfrak{g}_{2} \oplus \mathfrak{f}_{4}\right)^{+++}$

\begin{tabular}{|c|c|c|c|c|c|c|c|c|c|c|c|c|}
\hline$l$ & $p_{r}$ & $p_{i}$ & \multicolumn{5}{|c|}{ vector $\alpha$} & $\alpha^{2}$ & $d_{r}$ & $d_{i}$ & $\mu$ & ind \\
\hline 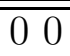 & $\begin{array}{lllll}0 & 0 & 0 & 1\end{array}$ & $\overline{000}$ & $\begin{array}{ccc}0 & 0 & 0\end{array}$ & 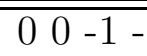 & $1-1$ & $\overline{1}$ & $\overline{-1}$ & $\overline{12}$ & 35 & $\overline{1}$ & 1 & $\overline{6}$ \\
\hline 00 & 0000 & 010 & $-3-20$ & $\begin{array}{lll}0 & 0 & 0\end{array}$ & 00 & 0 & $0 \quad 0$ & 12 & 1 & 14 & 7 & 0 \\
\hline 00 & $\begin{array}{lllll}0 & 0 & 0 & 0 & 0\end{array}$ & 002 & $\begin{array}{lll}0 & 0 & 0\end{array}$ & $\begin{array}{lll}-1 & 0 & 0\end{array}$ & 00 & 0 & $0 \quad 0$ & 6 & 1 & 3 & 1 & 0 \\
\hline 00 & $\begin{array}{llll}0 & 0 & 0 & 0\end{array}$ & 000 & $\begin{array}{lll}0 & 0 & 0\end{array}$ & $\begin{array}{lll}0 & 0 & 0\end{array}$ & 00 & 0 & $0 \quad 0$ & 0 & 1 & 1 & 1 & 0 \\
\hline 10 & $\begin{array}{llllll} & 0 & 0 & 1 & 0\end{array}$ & $\begin{array}{lll}0 & 1 & 0\end{array}$ & $\begin{array}{lll}0 & 0 & 1\end{array}$ & 000 & 00 & 0 & 00 & 12 & 15 & 14 & 1 & 4 \\
\hline 01 & 1000000 & 001 & $\begin{array}{lll}0 & 0 & 0\end{array}$ & $\begin{array}{lll}0 & 1 & 0\end{array}$ & $0 \quad$ & 0 & 00 & 6 & 6 & 2 & 1 & 1 \\
\hline 11 & $\begin{array}{llllll}0 & 0 & 0 & 0 & 1\end{array}$ & 011 & $\begin{array}{lll}0 & 0 & 1\end{array}$ & 011 & 11 & 1 & 10 & 6 & $\overline{6}$ & 28 & 1 & 5 \\
\hline 02 & $\begin{array}{llllll} & 1 & 1 & 0 & 0 & 0\end{array}$ & 002 & $\begin{array}{lll}0 & 0 & 0\end{array}$ & 021 & 0 & $\overline{01}$ & 00 & 12 & 15 & 3 & 1 & 2 \\
\hline 12 & $\begin{array}{lllll}1 & 0 & 0 & 0 & 1\end{array}$ & 012 & $\begin{array}{lll}0 & 0 & 1\end{array}$ & $\begin{array}{lll}02 & 1\end{array}$ & 11 & 1 & 10 & 12 & 35 & 42 & 1 & 6 \\
\hline 12 & $\begin{array}{lllll}0 & 0 & 0 & 0 & 0\end{array}$ & 012 & $\begin{array}{lll}0 & 0 & 1\end{array}$ & 022 & 22 & 2 & 21 & 0 & 1 & 42 & 1 & 6 \\
\hline 12 & 00000 & 010 & $\begin{array}{lll}0 & 0 & 1\end{array}$ & 122 & 2 & 22 & 21 & -6 & 1 & 14 & 1 & 6 \\
\hline 03 & $\begin{array}{llllll} & 0 & 0 & 1 & 0 & 0\end{array}$ & 001 & $\begin{array}{lll}0 & 0 & 0\end{array}$ & 132 & 11 & $0 \quad$ & 00 & 6 & 20 & 2 & 1 & 3 \\
\hline 04 & $\begin{array}{lllll}1 & 0 & 1 & 0 & 0\end{array}$ & 000 & $\begin{array}{lll}0 & 0 & 0\end{array}$ & 242 & 11 & $\overline{01}$ & 00 & 12 & 105 & 1 & 1 & 4 \\
\hline 04 & 00010 & 002 & $\begin{array}{lll}0 & 0 & 0\end{array}$ & 143 & 21 & 10 & $0 \quad 0$ & 6 & 15 & 3 & 1 & 4 \\
\hline 05 & $\begin{array}{llllll}1 & 0 & 0 & 1 & 0\end{array}$ & 001 & $\begin{array}{lll}0 & 0 & 0\end{array}$ & 253 & 21 & 11 & 00 & 6 & 84 & 2 & 1 & 5 \\
\hline 05 & $\begin{array}{lllll}0 & 0 & 0 & 0 & 1\end{array}$ & 003 & $\begin{array}{lll}0 & 0 & 0\end{array}$ & 154 & 32 & 21 & 10 & 6 & 6 & 4 & 1 & 5 \\
\hline 05 & $\begin{array}{lllll}0 & 0 & 0 & 0 & 1\end{array}$ & 001 & $\begin{array}{lll}0 & 0 & 0\end{array}$ & 254 & 32 & 21 & 10 & -6 & 6 & 2 & 1 & 5 \\
\hline 06 & $\begin{array}{llllll}0 & 1 & 0 & 1 & 0\end{array}$ & 002 & $\begin{array}{lll}0 & 0 & 0\end{array}$ & $\begin{array}{lll}26 & 4\end{array}$ & 2 & 11 & 00 & 12 & 189 & 3 & 1 & 6 \\
\hline 06 & $\begin{array}{lllll}1 & 0 & 0 & 0 & 1\end{array}$ & 002 & $\begin{array}{lll}0 & 0 & 0\end{array}$ & 264 & 3 & 2 & 10 & 0 & 35 & 3 & 1 & 6 \\
\hline 06 & $\begin{array}{lllll}10 & 0 & 0 & 1\end{array}$ & 000 & $\begin{array}{lll}0 & 0 & 0\end{array}$ & 364 & 3 & 2 & 10 & -6 & 35 & 1 & 2 & 6 \\
\hline 06 & $\begin{array}{lllll}0 & 0 & 0 & 0 & 0\end{array}$ & 004 & $\begin{array}{lll}0 & 0 & 0\end{array}$ & 165 & 4 & 32 & 21 & 6 & 1 & 5 & 1 & 0 \\
\hline 06 & 00000 & 002 & $0 \quad 00$ & 265 & 4 & 32 & 21 & -12 & 1 & 3 & 2 & 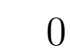 \\
\hline
\end{tabular}

Table 7: $\mathfrak{s l}(6)_{r} \oplus\left(\mathfrak{g}_{2} \oplus \mathfrak{s l}(2)\right)_{i}$ representations in $\left(\mathfrak{g}_{2} \oplus\right.$ $\left.\mathfrak{f}_{4}\right)^{+++}$ 


\begin{tabular}{|c|c|c|c|c|c|c|c|c|}
\hline & $p_{r}$ & $p_{i}$ & vector $\alpha$ & $\alpha^{2}$ & $d_{r}$ & $d_{i}$ & $\mu$ & ind \\
\hline 0 & $\begin{array}{llll}1 & 0 & 0 & 1\end{array}$ & & $-1-1-1-1$ & 6 & $\overline{24}$ & $\overline{00}$ & 1 & $\overline{5}$ \\
\hline 0 & $\begin{array}{llll}0 & 0 & 0 & 0\end{array}$ & & $\begin{array}{lllll}0 & 0 & 0 & 0 & 0 \\
\end{array}$ & 0 & 1 & 0 & 1 & 0 \\
\hline 1 & 10000 & & \begin{tabular}{lllll|}
1 & 0 & 0 & 0 & 0
\end{tabular} & 2 & 5 & 0 & 1 & 1 \\
\hline 2 & 0100 & & \begin{tabular}{lllll|}
2 & 1 & 0 & 0 & 0
\end{tabular} & 2 & 10 & 0 & 1 & 2 \\
\hline 3 & 1100 & & $\begin{array}{|lllll|}3 & 1 & 0 & 0 & 0 \\
\end{array}$ & 6 & 40 & 0 & 1 & 3 \\
\hline 4 & 1010 & & $\begin{array}{|lllll|}4 & 2 & 1 & 0 & 0 \\
\end{array}$ & 2 & 45 & 0 & 1 & $\overline{4}$ \\
\hline 5 & 01110 & & $\begin{array}{|lllll|}5 & 3 & 1 & 0 & 0\end{array}$ & 2 & 75 & 0 & 1 & 5 \\
\hline 5 & $\begin{array}{llll}10 & 0 & 1\end{array}$ & & $\begin{array}{lllll}5 & 3 & 2 & 1 & 0\end{array}$ & -4 & 24 & 0 & 1 & 5 \\
\hline
\end{tabular}

Table 8: $\mathfrak{s l}(5)_{r}$ representations in $\mathfrak{g}_{2}^{+}$

\begin{tabular}{|c|c|c|c|c|c|c|c|c|}
\hline$l$ & $p_{r}$ & $p_{i}$ & vector $\alpha$ & $\alpha^{2}$ & $d_{r}$ & $d_{i}$ & $\mu$ & ind \\
\hline 00 & $\begin{array}{lll}0 & 0 & 1\end{array}$ & 0 & $\begin{array}{llllllll} & 0 & 0 & -1 & -1 & -1 & -1\end{array}$ & 6 & 24 & 1 & 1 & 5 \\
\hline 00 & 0000 & 2 & $\begin{array}{lllllll}-1 & 0 & 0 & 0 & 0 & 0 & 0\end{array}$ & 6 & 1 & 3 & 1 & 0 \\
\hline 00 & 0000 & 0 & $\begin{array}{lllllll}0 & 0 & 0 & 0 & 0 & 0 & 0 \\
\end{array}$ & 0 & 1 & 1 & 1 & 0 \\
\hline 10 & 0010 & 2 & $\begin{array}{lllllll}0 & 1 & 0 & 0 & 0 & 0 & 0\end{array}$ & 6 & 10 & 3 & 1 & 3 \\
\hline 01 & 1000 & 0 & $\begin{array}{lllllll}0 & 0 & 1 & 0 & 0 & 0 & 0\end{array}$ & 2 & 5 & 1 & 1 & 1 \\
\hline 11 & 00001 & 2 & $\begin{array}{llllllll} & 1 & 1 & 1 & 1 & 1 & 0 \\
\end{array}$ & 2 & 5 & 3 & 1 & 4 \\
\hline 02 & 0100 & 0 & $\begin{array}{lllllll} & 0 & 2 & 1 & 0 & 0 & 0\end{array}$ & 2 & 10 & 1 & 1 & 2 \\
\hline 12 & 10001 & 2 & $\begin{array}{llllllll}0 & 1 & 2 & 1 & 1 & 1 & 0\end{array}$ & 2 & 24 & 3 & 1 & 5 \\
\hline 12 & 0000 & 2 & $\begin{array}{lllllll}0 & 1 & 2 & 2 & 2 & 2 & 1\end{array}$ & -4 & 1 & 3 & 1 & 5 \\
\hline 03 & 1100 & 0 & $\begin{array}{llllllll}0 & 0 & 3 & 1 & 0 & 0 & 0 \\
\end{array}$ & 6 & 40 & 1 & 1 & 3 \\
\hline 04 & 1010 & 0 & $\begin{array}{lllllll}0 & 0 & 4 & 2 & 1 & 0 & 0 \\
\end{array}$ & 2 & 45 & 1 & 1 & 4 \\
\hline 05 & 0110 & 0 & $\begin{array}{lllllll}0 & 0 & 5 & 3 & 1 & 0 & 0\end{array}$ & 2 & 75 & 1 & 1 & 5 \\
\hline 05 & 1001 & 0 & $\begin{array}{lllllll}0 & 0 & 5 & 3 & 2 & 1 & 0\end{array}$ & -4 & 24 & 1 & 1 & 5 \\
\hline
\end{tabular}

Table 9: $\mathfrak{s l}(5)_{r} \oplus \mathfrak{s u}(2)_{i}$ representations in $\left(\mathfrak{g}_{2} \oplus \mathfrak{s u}(2)\right)^{+++}$

\begin{tabular}{|c|c|c|c|c|c|c|c|c|}
\hline$l$ & $p_{r}$ & $p_{i}$ & vector $\alpha$ & $\alpha^{2}$ & $d_{r}$ & $d_{i}$ & $\mu$ & $\overline{\text { ind }}$ \\
\hline 00 & 00 & 001 & $\begin{array}{llllllll}0 & 0 & -3 & -2 & 0 & 0 & 0\end{array}$ & 6 & 1 & 14 & 1 & $\overline{0}$ \\
\hline 00 & 11 & 000 & $\begin{array}{llllllll}0 & 0 & 0 & 0 & 0 & -1 & -1\end{array}$ & 6 & 8 & 1 & 1 & 3 \\
\hline 00 & 00 & 200 & -100000000 & 6 & 1 & 3 & 1 & 0 \\
\hline 00 & 00 & 000 & $\begin{array}{lllllll}0 & 0 & 0 & 0 & 0 & 0 & 0\end{array}$ & 0 & 1 & 1 & 2 & 0 \\
\hline 10 & 10 & 200 & $\begin{array}{lllllll}0 & 1 & 0 & 0 & 0 & 0 & 0\end{array}$ & 6 & 3 & 3 & 1 & 1 \\
\hline 01 & 10 & 001 & $\begin{array}{llllllll}0 & 0 & 0 & 0 & 1 & 0 & 0 \\
\end{array}$ & 6 & 3 & 14 & 1 & 1 \\
\hline 11 & 01 & 201 & $\begin{array}{llllllll}0 & 1 & 0 & 0 & 1 & 1 & 0 \\
\end{array}$ & 6 & 3 & 42 & 1 & 2 \\
\hline 20 & 20 & 200 & $\begin{array}{lllllll}1 & 2 & 0 & 0 & 0 & 0 & 0\end{array}$ & 6 & 6 & 3 & 1 & 2 \\
\hline 20 & 01 & 000 & $\begin{array}{lllllll}2 & 2 & 0 & 0 & 0 & 1 & 0\end{array}$ & -6 & 3 & 1 & 1 & 2 \\
\hline 02 & 20 & 001 & $\begin{array}{lllllll}0 & 0 & 3 & 2 & 2 & 0 & 0\end{array}$ & 6 & 6 & 14 & 1 & 2 \\
\hline 02 & 01 & 020 & $\begin{array}{llllllll}0 & 0 & 2 & 2 & 2 & 1 & 0\end{array}$ & 2 & 3 & 27 & 1 & 2 \\
\hline 02 & 01 & 000 & $\begin{array}{lllllll}0 & 0 & 6 & 4 & 2 & 1 & 0 \\
\end{array}$ & -6 & 3 & 1 & 1 & 2 \\
\hline
\end{tabular}




\begin{tabular}{|rr|ll|lll|lllllll|r|r|r|r|r|}
\hline 2 & 1 & 1 & 1 & 2 & 0 & 1 & 1 & 2 & 0 & 0 & 1 & 1 & 0 & 0 & 8 & 42 & 1 & 3 \\
2 & 1 & 1 & 1 & 0 & 0 & 1 & 2 & 2 & 0 & 0 & 1 & 1 & 0 & -6 & 8 & 14 & 1 & 3 \\
2 & 1 & 0 & 0 & 2 & 0 & 1 & 1 & 2 & 0 & 0 & 1 & 2 & 1 & -6 & 1 & 42 & 1 & 3 \\
2 & 1 & 0 & 0 & 0 & 0 & 1 & 2 & 2 & 0 & 0 & 1 & 2 & 1 & -12 & 1 & 14 & 1 & 3 \\
\hline 1 & 2 & 0 & 0 & 2 & 3 & 0 & 0 & 1 & 0 & 1 & 2 & 2 & 1 & 6 & 1 & 231 & 1 & 3 \\
1 & 2 & 1 & 1 & 2 & 2 & 0 & 0 & 1 & 2 & 2 & 2 & 1 & 0 & 2 & 8 & 81 & 1 & 3 \\
1 & 2 & 1 & 1 & 2 & 0 & 1 & 0 & 1 & 3 & 2 & 2 & 1 & 0 & 0 & 8 & 42 & 1 & 3 \\
1 & 2 & 0 & 0 & 2 & 2 & 0 & 0 & 1 & 2 & 2 & 2 & 2 & 1 & -4 & 1 & 81 & 1 & 3 \\
1 & 2 & 0 & 0 & 2 & 0 & 1 & 0 & 1 & 3 & 2 & 2 & 2 & 1 & -6 & 1 & 42 & 1 & 3 \\
1 & 2 & 1 & 1 & 2 & 0 & 0 & 0 & 1 & 6 & 4 & 2 & 1 & 0 & -6 & 8 & 3 & 1 & 3 \\
1 & 2 & 0 & 0 & 2 & 0 & 0 & 0 & 1 & 6 & 4 & 2 & 2 & 1 & -12 & 1 & 3 & 1 & 3 \\
\hline 3 & 0 & 3 & 0 & 2 & 0 & 0 & 2 & 3 & 0 & 0 & 0 & 0 & 0 & 6 & 10 & 3 & 1 & 3 \\
3 & 0 & 1 & 1 & 2 & 0 & 0 & 2 & 3 & 0 & 0 & 0 & 1 & 0 & -6 & 8 & 3 & 1 & 3 \\
3 & 0 & 1 & 1 & 0 & 0 & 0 & 3 & 3 & 0 & 0 & 0 & 1 & 0 & -12 & 8 & 1 & 1 & 3 \\
\hline 0 & 3 & 1 & 1 & 0 & 3 & 0 & 0 & 0 & 3 & 3 & 3 & 1 & 0 & 6 & 8 & 77 & 1 & 3 \\
0 & 3 & 3 & 0 & 0 & 0 & 1 & 0 & 0 & 6 & 4 & 3 & 0 & 0 & 6 & 10 & 14 & 1 & 3 \\
0 & 3 & 0 & 0 & 0 & 1 & 1 & 0 & 0 & 4 & 3 & 3 & 2 & 1 & -4 & 1 & 64 & 1 & 3 \\
0 & 3 & 1 & 1 & 0 & 2 & 0 & 0 & 0 & 5 & 4 & 3 & 1 & 0 & -4 & 8 & 27 & 1 & 3 \\
0 & 3 & 1 & 1 & 0 & 0 & 1 & 0 & 0 & 6 & 4 & 3 & 1 & 0 & -6 & 8 & 14 & 1 & 3 \\
0 & 3 & 0 & 0 & 0 & 2 & 0 & 0 & 0 & 5 & 4 & 3 & 2 & 1 & -10 & 1 & 27 & 1 & 3 \\
0 & 3 & 0 & 0 & 0 & 0 & 1 & 0 & 0 & 6 & 4 & 3 & 2 & 1 & -12 & 1 & 14 & 1 & 3 \\
0 & 3 & 1 & 1 & 0 & 1 & 0 & 0 & 0 & 7 & 5 & 3 & 1 & 0 & -10 & 8 & 7 & 1 & 3 \\
0 & 3 & 1 & 1 & 0 & 0 & 0 & 0 & 0 & 9 & 6 & 3 & 1 & 0 & -12 & 8 & 1 & 1 & 3 \\
\hline
\end{tabular}

Table 10: $\mathfrak{s l}(3)_{r} \oplus\left(\mathfrak{g}_{2} \oplus \mathfrak{s u}(2)\right)_{i}$ representations in $\left(\mathfrak{g}_{2} \oplus\right.$ $\mathfrak{s u}(2))^{+++}$

\begin{tabular}{|c|c|c|c|c|c|c|c|c|}
\hline$l$ & $p_{r}$ & $p_{i}$ & vector $\alpha$ & $\alpha^{2}$ & $d_{r}$ & $d_{i}$ & $\mu$ & ind \\
\hline 00 & 101 & $\overline{0}$ & $\begin{array}{llllllll}0 & 0 & 0 & -1 & -1 & -1\end{array}$ & 2 & 15 & $\overline{1}$ & 1 & 4 \\
\hline 00 & 000 & 2 & $\begin{array}{llllll}-1 & 0 & 0 & 0 & 0 & 0\end{array}$ & 2 & 1 & 3 & 1 & 0 \\
\hline 00 & 000 & 0 & $\begin{array}{llllll}0 & 0 & 0 & 0 & 0 & 0\end{array}$ & 0 & 1 & 1 & 1 & 0 \\
\hline 10 & 010 & 2 & $\begin{array}{llllll}0 & 1 & 0 & 0 & 0 & 0\end{array}$ & 2 & 6 & 3 & 1 & 2 \\
\hline 01 & 200 & 0 & $\begin{array}{llllllll}0 & 0 & 1 & 0 & 0 & 0 \\
\end{array}$ & 2 & 10 & 1 & 1 & 2 \\
\hline 11 & 101 & 2 & $\begin{array}{lllllll}0 & 1 & 1 & 1 & 1 & 0\end{array}$ & 0 & 15 & 3 & 1 & 4 \\
\hline 20 & 020 & 2 & $\begin{array}{lllllll}12 & 0 & 0 & 0 & 0\end{array}$ & 2 & 20 & 3 & 1 & 4 \\
\hline 20 & 101 & 0 & $\begin{array}{llllll}2 & 2 & 0 & 0 & 1 & 0\end{array}$ & -2 & 15 & 1 & 1 & 4 \\
\hline 20 & 000 & 2 & $\begin{array}{llllll}1 & 2 & 0 & 1 & 2 & 1 \\
\end{array}$ & -2 & 1 & 3 & 1 & 4 \\
\hline 02 & 210 & 0 & $\begin{array}{lllllll}0 & 0 & 2 & 1 & 0 & 0 \\
\end{array}$ & 2 & 45 & 1 & 1 & 4 \\
\hline
\end{tabular}

Table 11: $\quad \mathfrak{s l}(4)_{r} \oplus \mathfrak{s l}(2)_{i}$ representations in $(\mathfrak{s l}(2) \oplus$ $\mathfrak{s l}(2))^{+++}$ 


\begin{tabular}{|c|c|c|c|c|c|c|c|c|}
\hline$l$ & $p_{r}$ & $p_{i}$ & vector $\alpha$ & $\alpha^{2}$ & $d_{r}$ & $d_{i}$ & $\mu$ & ind \\
\hline$\overline{000}$ & 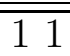 & 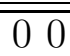 & $\begin{array}{ccccccc}0 & 0 & 0 & 0 & -1 & -1\end{array}$ & 2 & 8 & 1 & $\overline{\overline{1}}$ & $\overline{\overline{3}}$ \\
\hline 00 & 00 & 02 & $\begin{array}{lllllll}0 & 0 & -1 & 0 & 0 & 0\end{array}$ & 2 & 1 & 3 & & \\
\hline 00 & 0 & 20 & $\begin{array}{llllll}-1 & 0 & 0 & 0 & 0 & 0\end{array}$ & 2 & 1 & 3 & & \\
\hline 00 & 00 & 00 & $\begin{array}{lllllll}0 & 0 & 0 & 0 & 0 & 0\end{array}$ & 0 & 1 & 1 & 1 & 0 \\
\hline 10 & 10 & 20 & $\begin{array}{lllllll}0 & 1 & 0 & 0 & 0 & 0\end{array}$ & 2 & 3 & 3 & 1 & 1 \\
\hline 01 & 10 & 02 & $\begin{array}{lllllll}0 & 0 & 0 & 1 & 0 & 0\end{array}$ & 2 & 3 & 3 & 1 & 1 \\
\hline 11 & 01 & 22 & $\begin{array}{lllllll}0 & 1 & 0 & 1 & 1 & 0\end{array}$ & 2 & 3 & 9 & 1 & 2 \\
\hline 20 & 20 & 20 & $\begin{array}{lllllll}1 & 2 & 0 & 0 & 0 & 0\end{array}$ & 2 & 6 & 3 & 1 & 2 \\
\hline 20 & 01 & 00 & $\begin{array}{lllllll}2 & 2 & 0 & 0 & 1 & 0\end{array}$ & -2 & 3 & 1 & 1 & 2 \\
\hline 02 & 20 & 02 & $\begin{array}{lllllll}0 & 0 & 1 & 2 & 0 & 0\end{array}$ & 2 & 6 & 3 & 1 & 2 \\
\hline 02 & 01 & 00 & $\begin{array}{lllllll}0 & 0 & 2 & 2 & 1 & 0\end{array}$ & -2 & 3 & 1 & 1 & 2 \\
\hline 21 & 11 & 22 & $\begin{array}{lllllll}1 & 2 & 0 & 1 & 1 & 0\end{array}$ & 0 & 8 & 9 & 1 & 3 \\
\hline 21 & 11 & 02 & $\begin{array}{lllllll}2 & 2 & 0 & 1 & 1 & 0\end{array}$ & -2 & 8 & 3 & 1 & 3 \\
\hline 21 & 00 & 22 & $\begin{array}{llllll}1 & 2 & 0 & 1 & 2 & 1\end{array}$ & -2 & 1 & 9 & 1 & 3 \\
\hline 21 & 00 & 02 & $\begin{array}{llllll}2 & 2 & 0 & 1 & 2 & 1\end{array}$ & -4 & 1 & 3 & 1 & 3 \\
\hline 12 & 11 & 22 & $\begin{array}{lllllll}0 & 1 & 1 & 2 & 1 & 0\end{array}$ & $\overline{0}$ & 8 & 9 & 1 & 3 \\
\hline 12 & 11 & 20 & $\begin{array}{lllllll}0 & 1 & 2 & 2 & 1 & 0\end{array}$ & -2 & 8 & 3 & 1 & 3 \\
\hline 12 & 00 & 22 & $\begin{array}{llllll}0 & 1 & 1 & 2 & 2 & 1\end{array}$ & -2 & 1 & 9 & 1 & 3 \\
\hline 12 & 00 & 20 & $\begin{array}{llllll}0 & 1 & 2 & 2 & 2 & 1\end{array}$ & -4 & 1 & 3 & 1 & 3 \\
\hline 30 & 30 & 20 & $\begin{array}{llllll}2 & 3 & 0 & 0 & 0 & 0\end{array}$ & 2 & 10 & 3 & $\overline{1}$ & 3 \\
\hline 30 & 11 & 20 & $\begin{array}{lllllll}2 & 3 & 0 & 0 & 1 & 0\end{array}$ & -2 & 8 & 3 & 1 & 3 \\
\hline 30 & 11 & 00 & $\begin{array}{llllll}3 & 3 & 0 & 0 & 1 & 0\end{array}$ & -4 & 8 & 1 & 1 & 3 \\
\hline 03 & 30 & 02 & $\begin{array}{lllllll}0 & 0 & 2 & 3 & 0 & 0\end{array}$ & 2 & 10 & 3 & $\overline{1}$ & 3 \\
\hline 03 & 11 & 02 & $\begin{array}{lllllll}0 & 0 & 2 & 3 & 1 & 0\end{array}$ & -2 & 8 & 3 & 1 & 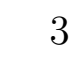 \\
\hline 03 & 11 & 00 & $\begin{array}{lllllll}0 & 0 & 3 & 3 & 1 & 0\end{array}$ & -4 & 8 & 1 & 1 & 3 \\
\hline
\end{tabular}

Table 12: $\mathfrak{s l}(3)_{r} \oplus \mathfrak{s l}(2)_{i} \oplus \mathfrak{s l}(2)_{i}$ representations in $(\mathfrak{s l}(2) \oplus$ $\mathfrak{s l}(2))^{+++}$

\section{References}

[1] E. Cremmer and B. Julia, Phys. Lett. B80 (1978) 48.

[2] B. Julia, LPTENS-80-16.

[3] B. Julia, in: Vertex Operators in Mathematics and Physics, Publications of the Mathematical Sciences Research Institute no 3, Springer Verlag (1984).

[4] R. Geroch, J. Math. Phys. 13, 394 (1972).

[5] P. Breitenlohner and D. Maison, Annales Poincare Phys. Theor. 46 (1987) 215.

[6] H. Nicolai, Phys. Lett. B 194 (1987) 402. 
[7] H. Nicolai, Phys. Lett. B 276 (1992) 333.

[8] T. Damour, M. Henneaux and H. Nicolai, Phys. Rev. Lett. 89 (2002) 221601 arXiv:hep-th/0207267.

[9] T. Damour and H. Nicolai, arXiv:hep-th/0410245.

[10] A. Kleinschmidt and H. Nicolai, Phys. Lett. B $606 \quad$ (2005) 391 arXiv:hep-th/0411225].

[11] P. C. West, Class. Quant. Grav. 18 (2001) 4443 [arXiv:hep-th/0104081].

[12] P. West, Phys. Lett. B 575 (2003) 333 arXiv:hep-th/0307098.

[13] I. Schnakenburg and P. C. West, Phys. Lett. B $517 \quad$ (2001) 421 arXiv:hep-th/0107181.

[14] P. West, Class. Quant. Grav. 20 (2003) 2393 arXiv:hep-th/0212291.

[15] H. Nicolai and T. Fischbacher, arXiv:hep-th/0301017.

[16] A. Kleinschmidt, I. Schnakenburg and P. West, Class. Quant. Grav. 21 (2004) 2493 arXiv:hep-th/0309198.

[17] F. Riccioni, A. Van Proeyen and P. West, arXiv:0801.2763 [hep-th].

[18] F. Riccioni and P. West, JHEP 0707, 063 (2007) [arXiv:0705.0752 [hep-th]].

[19] E. A. Bergshoeff, I. De Baetselier and T. A. Nutma, JHEP 0709, 047 (2007) [arXiv:0705.1304 [hep-th]].

[20] E. A. Bergshoeff, J. Gomis, T. A. Nutma and D. Roest, JHEP 0802, 069 (2008) arXiv:0711.2035 [hep-th]].

[21] E. A. Bergshoeff, M. de Roo, S. F. Kerstan and F. Riccioni, JHEP 0508 (2005) 098 arXiv:hep-th/0506013].

[22] P. West, JHEP 0603 (2006) 072 arXiv:hep-th/0511153].

[23] F. Riccioni and P. West, JHEP 0802 (2008) 039 [arXiv:0712.1795 [hep-th]].

[24] E. A. Bergshoeff, O. Hohm and T. A. Nutma, arXiv:0803.2989 [hep-th].

[25] B. de Wit, A. K. Tollsten and H. Nicolai, Nucl. Phys. B 392 (1993) 3 arXiv:hep-th/9208074].

[26] M. Gunaydin, G. Sierra and P. K. Townsend, Phys. Lett. B 133 (1983) 72.

[27] P. Goddard and D. Olive, in: J. Lepowsky, S. Mandelstam and I. M. Singer (eds.), Vertex Operators in Mathematics and Physics, Springer-Verlag (New York, 1985) 51-96. 
[28] M. R. Gaberdiel, D. I. Olive and P. C. West, Nucl. Phys. B 645 (2002) 403 arXiv:hep-th/0205068.

[29] B. de Wit and A. Van Proeyen, arXiv:hep-th/9505097.

[30] J. A. Strathdee, Int. J. Mod. Phys. A 2 (1987) 273.

[31] A. Keurentjes, Nucl. Phys. B 658 (2003) 303 arXiv:hep-th/0210178.

[32] H. Nicolai and H. Samtleben, Q. J. Pure Appl. Math. 1 (2005) 180 arXiv:hep-th/0407055.

[33] T. Damour, A. Kleinschmidt and H. Nicolai, Phys. Lett. B 634 (2006) 319 arXiv:hep-th/0512163.

[34] S. de Buyl, M. Henneaux and L. Paulot, JHEP 0602 (2006) 056 arXiv:hep-th/0512292.

[35] A. Kleinschmidt, arXiv:hep-th/0703262.

[36] J. Gomis and D. Roest, JHEP 0711, 038 (2007) arXiv:0706.0667 [hep-th]].

[37] E. Cremmer, LPTENS-80-17.

[38] S. Mizoguchi, K. Mohri and Y. Yamada, Class. Quant. Grav. 23 (2006) 3181 arXiv:hep-th/0512092.

[39] V. Kac, Infinite dimensional Lie algebras (Cambridge University Press, Cambridge 1990).

[40] T. Damour and M. Henneaux, Phys. Rev. Lett. $85 \quad$ (2000) 920 arXiv:hep-th/0003139.

[41] T. Damour, M. Henneaux, B. Julia and H. Nicolai, Phys. Lett. B 509 (2001) 323 arXiv:hep-th/0103094.

[42] T. Damour, S. de Buyl, M. Henneaux and C. Schomblond, JHEP 0208 (2002) 030 arXiv:hep-th/0206125.

[43] H. Nicolai, Schladming lectures, DESY 91-038.

[44] R. Geroch, J. Math. Phys. 12 (1971) 918.

[45] B. Julia, LPTENS-81-14.

[46] B. Julia, LPTENS-82-23.

[47] V. Back-Valente, N. Bardy-Panse, H. Ben Messaoud, G. Rousseau, J. Algebra 171 (1995) 43-96.

[48] H. Ben Messaoud, J. Phys. A, Math. Gen. 39 (2006) 13659-13690. 
[49] N. A. Obers and B. Pioline, Phys. Rept. 318 (1999) 113 [arXiv:hep-th/9809039].

[50] D. V. Alekseevsky, Math. USSR Izvestija 9, 445 (1975) arXiv:hep-th/9112027.

[51] B. de Wit and A. Van Proeyen, Commun. Math. Phys. 149, 307 (1992) arXiv:hep-th/9112027.

[52] M. Gunaydin, L. J. Romans and N. P. Warner, Nucl. Phys. B 272 (1986) 598.

[53] G. Dall'Agata, K. Lechner and M. Tonin, JHEP 9807 (1998) 017 [arXiv:hep-th/9806140].

[54] B. de Wit, I. Herger and H. Samtleben, Nucl. Phys. B 671 (2003) 175 arXiv:hep-th/0307006].

[55] E. Cremmer, B. Julia, H. Lu and C. N. Pope, arXiv:hep-th/9909099.

[56] M. Gunaydin, G. Sierra and P. K. Townsend, Nucl. Phys. B 253 (1985) 573.

[57] F. Riccioni, D. Steele and P. West, Class. Quant. Grav. 25 (2008) 045012 arXiv:0706.3659 [hep-th]].

[58] A. Keurentjes, Nucl. Phys. B 658, 348 (2003) [arXiv:hep-th/0212024].

[59] T. Fischbacher, JHEP 0508 (2005) 012 arXiv:hep-th/0504230].

[60] R. E. Borcherds, J. Algebra 115 (1988) 501-512.

[61] A. Kleinschmidt and H. Nicolai, JHEP 0407 (2004) 041 arXiv:hep-th/0407101].

[62] F. Riccioni and P. West, Phys. Lett. B 645 (2007) 286 arXiv:hep-th/0612001.

[63] D. Z. Freedman and A. K. Das, Nucl. Phys. B 120 (1977) 221.

[64] E. S. Fradkin and M. A. Vasiliev, LEBEDEV-76-197.

[65] J. Bagger and E. Witten, Phys. Lett. B 118 (1982) 103.

[66] B. Zumino, Phys. Lett. B 87 (1979) 203.

[67] J. A. Bagger, Nucl. Phys. B 211 (1983) 302.

[68] A. Van Proeyen, arXiv:hep-th/0301005.

[69] L. Andrianopoli, R. D'Auria and S. Ferrara, JHEP 0203 (2002) 025 arXiv:hep-th/0110277.

[70] A. Kleinschmidt and H. Nicolai, Phys. Lett. B 637 (2006) 107 arXiv:hep-th/0603205.

[71] C. Hillmann and A. Kleinschmidt, Gen. Rel. Grav. 38 (2006) 1861 arXiv:hep-th/0608092.

[72] T. Nutma, SimpLie, http://strings.fmns.rug.nl/SimpLie//. 